\title{
Sliding Mode Control Design Using Canonical Homogeneous Norm
}

\author{
Andrey Polyakov*
}

\begin{abstract}
The problem of sliding mode control design for nonlinear plant is studied. Necessary and sufficient conditions of quadratic-like stability (stabilizability) for nonlinear homogeneous (control) system are obtained. Sufficient conditions of robust stability/stabilizability are deduced. The results are supported with academic examples of sliding mode control design.
\end{abstract}

\section{Introduction}

Symmetry is one of well-known properties of physical systems [1]. A certain form of symmetry studied in systems and control theory is known as homogeneity [2], [3], [4], [5], [6]. The standard homogeneity introduced by L. Euler in 17th century is the symmetry of a mathematical object $f$ (e.g. function, vector field, operator, etc) with respect to the uniform dilation of the argument $x \rightarrow \lambda x$, namely, $f(\lambda x)=\lambda f(x), \lambda>0$. Type of homogeneity is basically identified by the corresponding dilation. For example, in [7], [8], [9] the uniform dilation is utilized and the papers [2], [10], [11], [12] deal with the so-called weighted dilation. Nonlinear homogeneous differential equations/inclusions form an important class of control systems [4], [13], [14], [15], [16], [17]. They appear as local approximations [3], [15] or set-valued extensions [11], [12] of nonlinear systems and include models of process control [18], nonholonomic systems [19], mechanical models with frictions [11], etc.

The generalized homogeneity (to be studied in this paper) was introduced originally in [20] for infinite dimensional models such as partial differential equations (PDEs). It considers a strongly continuous group of linear bounded operators generated by a possibly unbounded linear operator as a dilation in a Banach space. A lot of well-known PDEs are homogeneous in generalized sense, e.g. heat, wave, Navier-Stocks, Saint-Venant, Korteweg-de Vries, fast diffusion equations. This paper deals with the finite-dimensional models of generalized homogeneous systems represented by ordinary differential equations (ODEs). It

\footnotetext{
*Non-A team, Inria Lille Nord Europe, 40 Avenue du Halley, 59650 Villeneuve-d'Ascq,
} France 
is worth stressing that all standard and weighted homogeneous systems are particular cases of the generalized homogeneous ones. Geometric homogeneity [21], [5], [4] is a more general type of symmetry allowing dilations to be nonlinear.

Stabilization of nonlinear plant is a classical control problem that is hard to solve constructively in general case, so particular solutions are still of the interest [22], [23]. Stability and stabilizability problems were studied for both standard [2], [9] and weighted homogeneous [10], [24], [25], [6], [26], [27], [28] systems which are the most popular today [11], [12], [14], [15], [17].

In the case of negative homogeneity degree the stability/stabilizability immediately implies finite-time stability/stabilizability [7], [29], [30], [11], [12], that is usually required for sliding mode control [31], [11], [12]. One of goals of this paper is to develop a universal sliding mode-like control design algorithm for an essentially nonlinear generalized homogeneous system.

This paper deals with quadratic stability (stabilizability) problem for nonlinear generalized homogeneous (control) systems. Quadratic stabilizability is useful property for control design, since the control tuning procedures in this case can be formalized as Linear Matrix Inequalities(LMIs) [32] and/or semidefinite programming problems [33], which are supported by MATLAB and other software.

First of all, we show that any generalized homogeneous system is diffeomorphic to a standard homogeneous one. Next, a change of coordinate, which transforms asymptotically stable generalized homogeneous system to a quadratically stable one is presented. In both cases the so-called canonical homogeneous norm [34] is utilized for construction of the corresponding coordinate transformation. Combination of these two results allows the necessary and sufficient stability condition to be presented in terms of existence of the quadratic-like Lyapunov function $V(x)=x^{\top} \Xi(x) P \Xi(x) x$, where $P=P^{\top} \succ 0$ and the nonsingular matrix $\Xi$ is constant along any ray from the origin (i.e. $\Xi\left(e^{s} x\right)=\Xi(x)$ for $x \neq 0, s \in \mathbb{R})$ and $\frac{\partial \Xi(x)}{\partial x_{i}} x=0$ for $x \neq 0$. The properties of matrix $\Xi$ motivate a conjecture about existence of quadratic Lyapunov function ( $\Xi=$ const), which fails in the general case. However, some examples show that the obtained stability condition may simplify finite-time stability/stabilizability analysis as well as sliding mode control design in some particular cases. As example, a class of high order sliding mode control allowing quadratic Lyapunov function is presented and an algorithm for selection of its parameters is given as LMIs.

Notation: $\mathbb{R}$ is the field of real numbers; $\mathbb{R}_{+}=\{x \in \mathbb{R}: x>0\} ; e_{i}=$ $(0, \ldots, 1, \ldots, 0)^{\top}$ is the identity vector in $\mathbb{R}^{n} ;\|\cdot\|$ denotes a norm in $\mathbb{R}^{n}$ and

$$
\|A\|_{\mathbb{A}}=\sup _{x \in \mathbb{R}^{n}} \frac{\|A x\|}{\|x\|} \quad \text { and } \quad\lfloor A\rfloor_{\mathbb{A}}=\inf _{x \in \mathbb{R}^{n}} \frac{\|A x\|}{\|x\|} \quad \text { if } \quad A \in \mathbb{R}^{n \times n} ;
$$

$C^{n}(X, Y)$ is the set of continuously differentiable (at least up to the order $n$ ) maps $X \rightarrow Y$, where $X, Y$ are open subsets of finite dimensional spaces; $\frac{\partial}{\partial x}=$ $\left(\frac{\partial}{\partial x_{1}}, \frac{\partial}{\partial x_{2}}, \ldots, \frac{\partial}{\partial x_{n}}\right) ; I_{n} \in \mathbb{R}^{n \times n}$ is the identity matrix; $\mathbf{0}$ denotes zero element, e.g. $\mathbf{0} \in \mathbb{R}^{n}$ is the zero vector but $\mathbf{0} \in \mathbb{R}^{n \times n}$ is the zero matrix; $\operatorname{diag}\left\{\lambda_{1}, . ., \lambda_{n}\right\}$ 
- diagonal matrix with elements $\lambda_{i}$; the order relation $P \succ 0$ means positive definiteness of the symmetric matrix $P \in \mathbb{R}^{n \times n} ; \lambda_{\max }(P)$ and $\lambda_{\min }(P)$ denote maximal and minimal eigenvalues of the symmetric matrix $P \in \mathbb{R}^{n \times n} ; \Re(\lambda)$ denotes the real part of the complex number $\lambda$; the notation $P^{\frac{1}{2}}$ means that $P^{\frac{1}{2}}=M$ such that $P=M^{2}$.

\section{Problem Statement}

It is well known [31] that sliding mode control design procedure consists of two steps. First, we select a sliding surface

$$
\sigma(q)=0,
$$

where $q \in \mathbb{R}^{r}$ is the state vector of a plant and $\sigma: \mathbb{R}^{r} \rightarrow \mathbb{R}^{n}$ is assumed to be sufficiently smooth. Next, we have to design a control that guarantees finite-time reaching of the surface $\sigma(q)=0$ and sliding on it.

Let us assume that the plant is modeled by a nonlinear ODE and the sliding surface is selected such that the differential equation describing the evolution of the sliding variable $\sigma(t)=\sigma(q(t))$ admits the representation

$$
\dot{\sigma}(t)=g(\sigma(t), u(t))+\omega(t, q(t), u(t)),
$$

where $t \in \mathbb{R}_{+}$is time variable, $\sigma(t) \in \mathbb{R}^{n}$ is the vector of sliding variables at the time instant $t, u(t) \in \mathbb{R}^{m}$ is the control input, $g \in C\left(\mathbb{R}^{n+m}, \mathbb{R}^{n}\right), g(\mathbf{0}, \mathbf{0})=\mathbf{0}$ and the function $\omega: \mathbb{R}^{r+m+1} \rightarrow \mathbb{R}^{n}$ is assumed to be unknown, but bounded in a certain way:

$$
\|\omega(t, q, u)\| \leq \bar{\omega}\left(\left\|\left(\begin{array}{c}
\sigma(q) \\
u
\end{array}\right)\right\|\right),
$$

where $\bar{\omega}: \mathbb{R}_{+} \rightarrow \mathbb{R}_{+}$is a continuous function. More constructive restrictions to $\bar{\omega}$ are given below (see, Section 6 ).

Therefore, sliding mode control design can interpreted as robust finite-time stabilization of the origin of the reduced-order system (1). In this paper we assume that only the vector of sliding variables $\sigma$ can be measured and utilized for control purposes. We deal (mainly) with the problem of a dynamical feedback control design

$$
\dot{u}(t)=k(\sigma(t), u(t)), \quad k \in C\left(\mathbb{R}^{n+m} \backslash\{\mathbf{0}\}, \mathbb{R}^{m}\right) .
$$

Finally, we restrict ourself with the class of the so-called generalized homogeneous nonlinear systems (see, [35] and the next section for more details).

The main goal of the paper is to derive a necessary and sufficient condition of finite-time stabilizability (with $\omega=0$ ) and a sufficient condition of robust finite-time stabilizability (for $\omega \neq 0$ ) of the system (1) by means of a homogeneous dynamical feedback (2). In particular, we are interested in a (more or less) constructive stabilizability criteria. Obviously, under assumption

$$
f=\left(\begin{array}{c}
g \\
k
\end{array}\right) \in C\left(\mathbb{R}^{n+m} \backslash\{\mathbf{0}\}, \mathbb{R}^{n+m}\right),
$$


the nominal closed-loop system (1), (2) may have discontinuity only at the origin similarly to the unit control [31] or "quasi-continuous" control [12]. It is also worth stressing that sliding mode control method frequently uses dynamical feedbacks as a part of the control law (see, e.g. super-twisting algorithm [36]).

\section{Generalized Homogeneity}

\subsection{Dilation Group}

Homogeneity is a sort of symmetry of an object (e.g. function or vector field) with respect to a group of transformations. The corresponding group is usually called group of dilations (or simply dilation). The generalized homogeneity [20] deals with the groups of linear transformations (linear dilations).

Definition 1 ([20]) A map $\boldsymbol{d}: \mathbb{R} \rightarrow \mathbb{R}^{n \times n}$ is called dilation in $\mathbb{R}^{n}$ if it satisfies

- Group property: $\boldsymbol{d}(0)=I_{n}$ and $\boldsymbol{d}(t+s)=\boldsymbol{d}(t) \boldsymbol{d}(s)=\boldsymbol{d}(s) \boldsymbol{d}(t), t, s \in \mathbb{R}$;

- Continuity property: $\boldsymbol{d}$ is a continuous map, i.e.

$$
\forall t>0, \forall \varepsilon>0, \exists \delta>0:|s-t|<\delta \Rightarrow\|\boldsymbol{d}(s)-\boldsymbol{d}(t)\|_{\mathbb{A}} \leq \varepsilon ;
$$

- Limit property: $\lim _{s \rightarrow-\infty}\|\boldsymbol{d}(s) x\|=0$ and $\lim _{s \rightarrow+\infty}\|\boldsymbol{d}(s) x\|=+\infty$ uniformly on the unit sphere

$$
S:=\left\{x \in \mathbb{R}^{n}:\|x\|=1\right\} .
$$

The dilation $\mathbf{d}$ is a continuous group of invertible linear maps $\mathbf{d}(s) \in \mathbb{R}^{n \times n}$, $\mathbf{d}(-s)=[\mathbf{d}(s)]^{-1}$. The matrix $G_{\mathbf{d}} \in \mathbb{R}^{n \times n}$ defined as

$$
G_{\mathbf{d}}=\lim _{s \rightarrow 0} \frac{\mathbf{d}(s)-I}{s}
$$

is known (see, e.g. [37, Ch. 1]) as the generator of the group d. It satisfies the following properties

$$
\frac{d}{d s} \mathbf{d}(s)=G_{\mathbf{d}} \mathbf{d}(s)=\mathbf{d}(s) G_{\mathbf{d}} \quad \text { and } \quad \mathbf{d}(s)=e^{G_{\mathbf{d}} s}:=\sum_{i=0}^{+\infty} \frac{s^{i} G_{\mathbf{d}}^{i}}{i !}, \quad s \in \mathbb{R} .
$$

The most popular dilations in $\mathbb{R}^{n}$ (see [13], [15], [12], [11], [14], [17] )

- uniform (or standard) dilation (L. Euler 17th century) :

$$
\mathbf{d}_{1}(s)=e^{s} I_{n}, \quad s \in \mathbb{R},
$$

- weighted dilation (Zubov 1958, [2]):

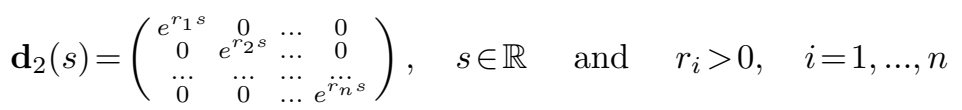

satisfy Definition 1 with $G_{\mathbf{d}_{1}}=I_{n}$ and $G_{\mathbf{d}_{2}}=\operatorname{diag}\left\{r_{i}\right\}$, respectively. Schematically the difference between uniform, weighted and generalized dilations is depicted at Figure 1. 


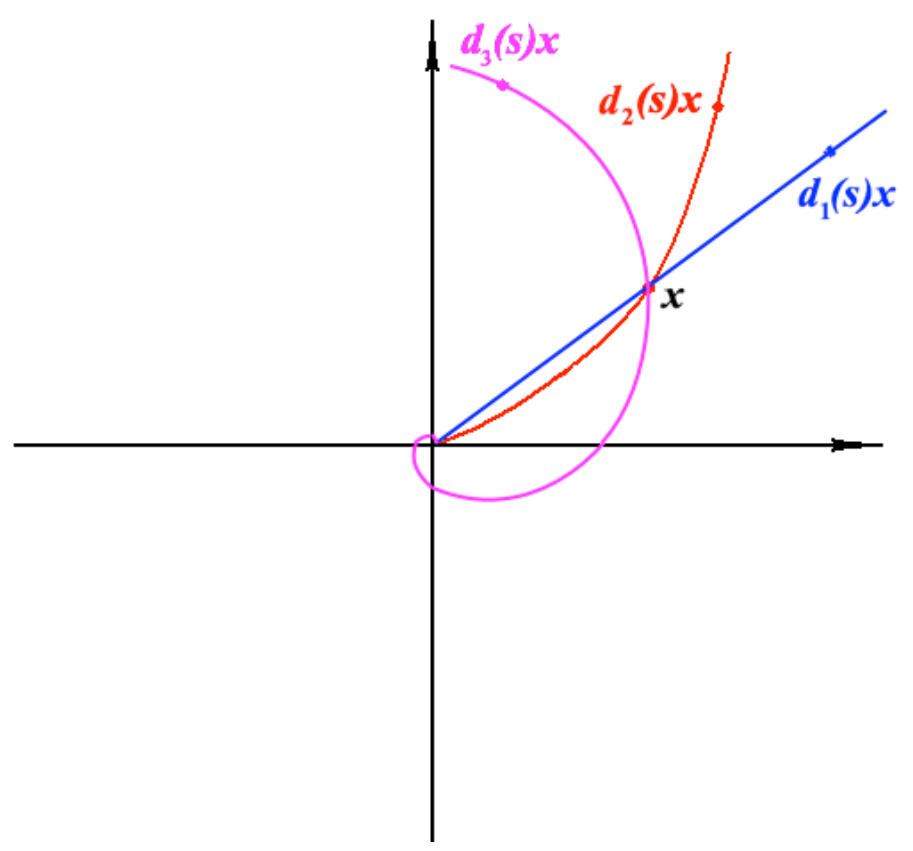

Figure 1: Illustration of uniform $\mathbf{d}_{1}$, weighted $\mathbf{d}_{2}$ and generalized $\mathbf{d}_{3}$ dilations

Definition 2 ([20]) The dilation $\boldsymbol{d}$ is said to be monotone if $\|\boldsymbol{d}(s)\|_{\mathbb{A}}<1$ as $s<0$.

Obviously, monotonicity of dilation may depend on a norm $\|\cdot\|$. For example, the dilation

$$
\mathbf{d}(s)=e^{s}\left(\begin{array}{cc}
\cos (s) & \sin (s) \\
-\sin (s) & \cos (s)
\end{array}\right) \text { with } G_{\mathbf{d}}=\left(\begin{array}{cc}
1 & 1 \\
-1 & 1
\end{array}\right)
$$

is monotone on $\mathbb{R}^{2}$ equipped with the weighted norm $\|x\|_{P}=\sqrt{x^{\top} P x}$ if $P=$ $\left(\begin{array}{cc}1 & 1 / \sqrt{2} \\ 1 / \sqrt{2} & 1\end{array}\right)>0$ and it is non-monotone if, for example, $P=\left(\begin{array}{cc}1 & 3 / 4 \\ 3 / 4 & 1\end{array}\right)>0$. In the latter case, the curve $\{\mathbf{d}(s) x: s \in \mathbb{R}\}$ may cross the unit sphere $S$ in two different points.

In other words, monotonicity of dilation means that the linear maps $\mathbf{d}(s)$ : $\mathbb{R}^{n} \rightarrow \mathbb{R}^{n}$ are strong contractions if $s<0$. Since $[\mathbf{d}(s)]^{-1}=\mathbf{d}(-s)$ then it is expectable that these maps are strong expansions for $s>0$.

Theorem 1 The next four conditions are equivalent

1) the dilation $\boldsymbol{d}$ is monotone;

2) $\lfloor d(s)\rfloor_{\mathbb{A}}>1$ for $s>0$;

3) the continuous function $\|\boldsymbol{d}(\cdot) x\|: \mathbb{R} \rightarrow \mathbb{R}_{+}$is strictly increasing for any fixed $x \in S$; 
4) for any $x \in \mathbb{R}^{n} \backslash\{\boldsymbol{O}\}$ there exists a unique pair $\left(s_{0}, x_{0}\right) \in \mathbb{R} \times S$ such that $x=\boldsymbol{d}\left(s_{0}\right) x_{0}$.

Proof. $\quad \mathbf{1}) \Rightarrow \mathbf{2})$ For any $x \in S$ we have $1=\|x\|=\|\mathbf{d}(-s) \mathbf{d}(s) x\| \leq$ $\|\mathbf{d}(-s)\|_{\mathbb{A}}\|\mathbf{d}(s) x\|$. Hence,

$$
1 \leq \inf _{x \in S}\|\mathbf{d}(-s)\|_{\mathbb{A}}\|\mathbf{d}(s) x\|=\|\mathbf{d}(-s)\|_{\mathbb{A}} \inf _{x \in S}\|\mathbf{d}(s) x\|=\|\mathbf{d}(-s)\|_{\mathbb{A}}\lfloor\mathbf{d}(s)\rfloor_{\mathbb{A}}
$$

for any $s \in \mathbb{R}$. The derived inequality gives $\lfloor\mathbf{d}(s)\rfloor_{\mathbb{A}} \geq \frac{1}{\|\mathbf{d}(-s)\|_{\mathbb{A}}}$. If the dilation is monotone then $\|\mathbf{d}(-s)\|_{\mathbb{A}}<1$ for $s>0$. So, we conclude $\lfloor\mathbf{d}(s)\rfloor_{\mathbb{A}}>1$ if $s>0$.

2) $\Rightarrow \mathbf{3}$ ) By definition of $\lfloor\cdot\rfloor_{\mathbb{A}}$ one has $\|\mathbf{d}(s) y\| \geq\lfloor\mathbf{d}(s)\rfloor_{\mathbb{A}}\|y\|$ for any $y \in \mathbb{R}^{n}$ and any $s \in \mathbb{R}$. If $s_{1}<s_{2}$ then $\left\lfloor\mathbf{d}\left(s_{2}-s_{1}\right)\right\rfloor_{\mathbb{A}}>1$ and for $x \neq 0$ we derive

$$
\begin{gathered}
\left\|\mathbf{d}\left(s_{1}\right) x\right\|-\left\|\mathbf{d}\left(s_{2}\right) x\right\|=\left\|\mathbf{d}\left(s_{1}\right) x\right\|-\left\|\mathbf{d}\left(s_{2}-s_{1}\right) \mathbf{d}\left(s_{1}\right) x\right\| \\
\leq\left(1-\left\lfloor\mathbf{d}\left(s_{2}-s_{1}\right)\right\rfloor_{\mathbb{A}}\right)\left\|\mathbf{d}\left(s_{1}\right) x\right\|<0 .
\end{gathered}
$$

The implication $\mathbf{3}) \Rightarrow \mathbf{4}$ ) is straightforward since $\|\mathbf{d}(0) x\|=\|x\|$. 4) $\Rightarrow \mathbf{1}$ ) If $x \in S$ then $\mathbf{d}(s) x \notin S$ for all $s \neq 0$. Indeed, otherwise the pair $\left(s_{0}, x_{0}\right) \in \mathbb{R} \times S$ such that $x_{0}=\mathbf{d}(s) x \in S$ is not unique. Hence, the limit property of the dilation (see, Definition 1) implies $\|\mathbf{d}(s) x\|<1$ for all $s<0$ and all $x \in S$.

Theorem 1 also proves that the functions $\|\mathbf{d}(\cdot)\|_{\mathbb{A}}: \mathbb{R} \rightarrow \mathbb{R}_{+}$and $[\mathbf{d}(\cdot)\rfloor_{\mathbb{A}}: \mathbb{R} \rightarrow \mathbb{R}_{+}$ are also continuous and strictly increasing.

Definition 3 ([34]) The dilation $\boldsymbol{d}$ is said to be strictly monotone on $\mathbb{R}^{n}$ if $\exists \beta>0$ such that $\|\boldsymbol{d}(s)\|_{\mathbb{A}} \leq e^{\beta s}$ for $s \leq 0$.

The dilation $\mathbf{d}$ considered in the above example is strictly monotone on $\mathbb{R}^{2}$ equipped with the conventional Euclidian norm.

Theorem 2 Let $\boldsymbol{d}$ be a dilation in $\mathbb{R}^{n}$ then

1) all eigenvalues $\lambda_{i}$ of the matrix $G_{\boldsymbol{d}}$ are placed in the right complex halfplane, i.e.

$$
\Re\left(\lambda_{i}\right)>0, \quad i=1,2, \ldots, n ;
$$

2) there exists a matrix $P \in \mathbb{R}^{n \times n}$ such that

$$
P G_{d}+G_{d}^{\top} P \succ 0, \quad P=P^{\top} \succ 0 ;
$$

3) the dilation $\boldsymbol{d}$ is strictly monotone with respect to the weighted Euclidean norm $\|\cdot\|=\sqrt{\langle\cdot, \cdot\rangle}$ induced by the inner product $\langle x, z\rangle=x^{\top} P z$ with $P$ satisfying (5):

$$
\begin{aligned}
& e^{\alpha s} \leq\lfloor\boldsymbol{d}(s)\rfloor_{\mathbb{A}} \leq\|\boldsymbol{d}(s)\|_{\mathbb{A}} \leq e^{\beta s} \quad \text { if } \quad s \leq 0, \\
& e^{\beta s} \leq\lfloor\boldsymbol{d}(s)\rfloor_{\mathbb{A}} \leq\|\boldsymbol{d}(s)\|_{\mathbb{A}} \leq e^{\alpha s} \quad \text { if } \quad s \geq 0,
\end{aligned}
$$


where

$$
\alpha=\frac{1}{2} \lambda_{\max }\left(P^{\frac{1}{2}} G_{d} P^{-\frac{1}{2}}+P^{-\frac{1}{2}} G_{d}^{\top} P^{\frac{1}{2}}\right)
$$

and

$$
\beta=\frac{1}{2} \lambda_{\min }\left(P^{\frac{1}{2}} G_{d} P^{-\frac{1}{2}}+P^{-\frac{1}{2}} G_{d}^{\top} P^{\frac{1}{2}}\right) .
$$

Proof. 1) Since $\frac{d}{d s} \mathbf{d}(s)=G_{\mathbf{d}} \mathbf{d}(s), \mathbf{d}(0)=I$ then $\mathbf{d}(s)=e^{s G_{\mathbf{d}}}=\sum_{i=0}^{+\infty} \frac{s^{i} G_{\mathbf{d}}^{i}}{i !}$ is the fundamental matrix of the linear system ODEs with the matrix $G_{\mathbf{d}}$. The limit property of the dilation implies that for all $x \neq \mathbf{0}$ one has $\left\|e^{s G_{\mathbf{d}}} x\right\| \rightarrow+\infty$ as $s \rightarrow+\infty$. This means that all eigenvalues $\lambda_{i}$ of the matrix $G_{\mathbf{d}}$ are placed in the right complex half-plane, i.e. the matrix $-G_{\mathbf{d}}$ is Hurwitz. Indeed, otherwise there exists an eigenvalue $\lambda_{*}: \Re\left(\lambda_{*}\right) \leq 0$ and there exists a non-trivial vector $x_{\lambda_{i *}}$ belonging to an eigensubspace of $\lambda_{*}$ such that $\left\|e^{s G_{\mathbf{d}}} x_{\lambda_{*}}\right\|$ is bounded for any $s \geq 0$. In particular, if $\lambda_{*}$ is a real eigenvalue of $G_{\mathbf{d}}$ and $x_{\lambda_{*}} \neq \mathbf{0}$ is a corresponding eigenvector then $G_{\mathbf{d}} x_{\lambda_{*}}=\lambda_{*} x_{\lambda_{*}}$ and

$$
e^{s G_{\mathbf{d}}} x_{\lambda_{*}}=\sum_{i=0}^{+\infty} \frac{s^{i} G_{\mathbf{d}}^{i}}{i !} x_{\lambda_{*}}=\sum_{i=0}^{+\infty} \frac{s^{i} \lambda_{*}^{i}}{i !} x_{\lambda_{*}}=e^{s \lambda_{*}} x_{\lambda_{*}} .
$$

Hence, if $\lambda_{*} \leq 0$ then $\left\|e^{s G_{\mathbf{d}}} x_{\lambda_{*}}\right\|=e^{s \lambda_{*}}\left\|x_{\lambda_{*}}\right\| \leq\left\|x_{\lambda_{*}}\right\|$ for all $s \geq 0$. This contradicts the limit property of the dilation. Similar constructions can be provided for the case of a complex conjugate pair of eigenvalues.

2) If $-G_{\mathbf{d}}$ is a Hurwitz matrix then for any positive definite matrix $Q=$ $Q^{\top} \in \mathbb{R}^{n \times n}$ the Lyapunov equation $P\left(-G_{\mathbf{d}}\right)+\left(-G_{\mathbf{d}}^{\top}\right) P=-Q$ has a unique positive definite solution $P=P^{\top} \in \mathbb{R}^{n \times n}$ (see, e.g. [32, Chapter 1]). Hence, $P G_{\mathbf{d}}+G_{\mathbf{d}}^{\top} P=Q \succ 0$, i.e. the linear matrix inequality (5) is always feasible provided that $G_{\mathbf{d}}$ is a generator of the dilation.

3) Recall that for any symmetric matrix $M=M^{\top} \in R^{n \times n}$ one has

$$
\lambda_{\min }(M) x^{\top} x \leq x^{\top} M x \leq \lambda_{\max }(M) x^{\top} x,
$$

but if, in addition, $M \succ 0$ then $N^{\top} M N \succ 0$ for any nonsingular matrix $N \in$ $\mathbb{R}^{n \times n}$ (see, e.g. [38]). Finally, taking into account

$$
\frac{d}{d s} \mathbf{d}(s)=\frac{d}{d s} e^{s G_{\mathbf{d}}}=G_{\mathbf{d}} e^{s G_{\mathbf{d}}}=e^{s G_{\mathbf{d}}} G_{\mathbf{d}}
$$

for $x \in \mathbb{R}^{n}$ we derive

$$
\begin{aligned}
\frac{d}{d s}\|\mathbf{d}(s) x\|^{2} & =\frac{d}{d s} x^{\top} \mathbf{d}(s)^{\top} P \mathbf{d}(s) x=x^{\top} \mathbf{d}(s)^{\top}\left(P G_{\mathbf{d}}+G_{\mathbf{d}}^{\top} P\right) \mathbf{d}(s) x \\
& =x^{\top} \mathbf{d}(s)^{\top} P^{\frac{1}{2}}\left(P^{\frac{1}{2}} G_{\mathbf{d}} P^{-\frac{1}{2}}+P^{-\frac{1}{2}} G_{\mathbf{d}}^{\top} P^{\frac{1}{2}}\right) P^{\frac{1}{2}} \mathbf{d}(s) x \\
& \geq 2 \beta x^{\top} \mathbf{d}(s)^{\top} P \mathbf{d}(s) x=2 \beta\|\mathbf{d}(s) x\|^{2} .
\end{aligned}
$$


Similarly we derive $\frac{d}{d s}\|\mathbf{d}(s) x\|^{2} \leq 2 \alpha\|\mathbf{d}(s) x\|^{2}$. Note that since $P \succ 0$ then $P^{\frac{1}{2}}=\left(P^{\frac{1}{2}}\right)^{\top} \succ 0$ (see, e.g [38]), but the matrix inequality (5) implies

$$
P^{\frac{1}{2}} G_{\mathbf{d}} P^{-\frac{1}{2}}+P^{-\frac{1}{2}} G_{\mathbf{d}}^{\top} P^{\frac{1}{2}} \succ 0
$$

and $0<\beta \leq \alpha$. Therefore, the obtained differential inequalities imply

$$
e^{2 \beta s}\|x\|^{2}=e^{2 \beta s}\|\mathbf{d}(0) x\|^{2} \leq\|\mathbf{d}(s) x\|^{2} \leq e^{2 \alpha s}\|\mathbf{d}(0) x\|^{2}=e^{2 \alpha s}\|x\|^{2} \quad \text { if } s \geq 0
$$

and

$$
e^{2 \alpha s}\|x\|^{2}=e^{2 \alpha s}\|\mathbf{d}(0) x\|^{2} \leq\|\mathbf{d}(s) x\|^{2} \leq e^{2 \beta s}\|\mathbf{d}(0) x\|^{2}=e^{2 \beta s}\|x\|^{2} \quad \text { if } s \leq 0 .
$$

or, equivalently, for $x \neq \mathbf{0}$ one has

$$
e^{\beta s} \leq \frac{\|\mathbf{d}(s) x\|}{\|x\|} \leq e^{\alpha s} \quad \text { if } s \geq 0 \quad \text { and } \quad e^{\alpha s} \leq \frac{\|\mathbf{d}(s) x\|}{\|x\|} \leq e^{\beta s} \quad \text { if } s \geq 0
$$

Hence, taking the supremum (resp. infinum) on $x \neq 0$ in the latter inequalities we conclude that the inequalities for matrix norms hold.

The latter theorem proves that any dilation $\boldsymbol{d}$ is strictly monotone if $\mathbb{R}^{n}$ is equipped with the weighted Euclidian norm $\|x\|=\sqrt{x^{\top} P x}$ provided that the matrix $P>0$ satisfies (5).

\subsection{Canonical Homogeneous Norm}

The dilation $\mathbf{d}$ introduces a sort of norm topology in $\mathbb{R}^{n}$ by means of the socalled "homogeneous norm" [4], [16], [15].

Definition 4 A continuous function $p: \mathbb{R}^{n} \rightarrow \mathbb{R}_{+}$is said to be $\boldsymbol{d}$-homogeneous norm if $p(x) \rightarrow 0$ as $x \rightarrow \mathbf{0}$ and $p(\boldsymbol{d}(s) x)=e^{s} p(x)>0$ for $x \in \mathbb{R}^{n} \backslash\{\mathbf{0}\}$ and $s \in \mathbb{R}$.

For monotone dilations the canonical homogeneous norm $\|\cdot\|_{\mathbf{d}}$ : $\mathbb{R}^{n} \rightarrow \mathbb{R}_{+}$is defined as

$$
\|x\|_{\mathbf{d}}=e^{s_{x}} \quad \text { where } \quad s_{x} \in \mathbb{R} \quad \text { such that } \quad\left\|\mathbf{d}\left(-s_{x}\right) x\right\|=1 .
$$

In [34] such a homogeneous norm was called canonical since it is induced by the canonical norm $\|\cdot\|$ in $\mathbb{R}^{n},\|x\|_{\mathbf{d}}=1$ if $\|x\|=1$ and, moreover, $\|\cdot\|_{\mathbf{d}}=\|\cdot\|$ if $\mathbf{d}(s)=e^{s} I_{n}$. Obviously, $\left\|\mathbf{d}\left(-\ln \|x\|_{\mathbf{d}}\right) x\right\|=1$ and

$$
\left\lfloor\mathbf{d}\left(\ln \|x\|_{\mathbf{d}}\right)\right\rfloor_{\mathbb{A}} \leq\|x\|=\left\|\mathbf{d}\left(\ln \|x\|_{\mathbf{d}}\right) \mathbf{d}\left(-\ln \|x\|_{\mathbf{d}}\right) x\right\| \leq\left\|\mathbf{d}\left(\ln \|x\|_{\mathbf{d}}\right)\right\|_{\mathbb{A}} .
$$

Proposition 1 If $\boldsymbol{d}$ is a strictly monotone dilation then 
- $\left|\left\|x_{1}\right\|_{d}^{\beta}-\left\|x_{2}\right\|_{d}^{\beta}\right| \leq\left\|x_{1}-x_{2}\right\|$ for $x_{i} \in \mathbb{R}^{n}:\left\|x_{i}\right\| \geq 1, i=1,2$, where $\beta$ is given in Definition 3;

- the homogeneous norm $\|\cdot\|_{d}$ is Lipschitz continuous outside the origin;

- if the norm $\|\cdot\|$ is smooth outside the origin then the homogeneous norm $\|\cdot\|_{\boldsymbol{d}}$ is also smooth outside the origin, $\frac{d\|\boldsymbol{d}(-s) x\|}{d s}<0$ if $s \in \mathbb{R}, x \in \mathbb{R}^{n} \backslash\{\mathbf{0}\}$ and

$$
\frac{\partial\|x\|_{d}}{\partial x}=\left.\frac{\left.\|x\|_{d} \frac{\partial\|z\|}{\partial z}\right|_{z=d(-s) x}}{\left.\frac{\partial\|z\|}{\partial z}\right|_{z=d(-s) x} G_{d} d(-s) x}\right|_{s=\ln \|x\|_{d}}
$$

Proof. Since for $x_{i} \in \mathbb{R}^{n}$ we have $\left\|x_{i}\right\|_{\mathbf{d}}=e^{s_{i}}:\left\|\mathbf{d}\left(-s_{i}\right) x_{i}\right\|=1$ then $1=\left\|\mathbf{d}\left(-s_{1}\right) x_{1}\right\|=\left\|\mathbf{d}\left(-s_{1}\right)\left(x_{1}-x_{2}\right)+\mathbf{d}\left(s_{2}-s_{1}\right) \mathbf{d}\left(-s_{2}\right) x_{2}\right\| \leq\left\|\mathbf{d}\left(-s_{1}\right)\right\|_{\mathbb{A}} \|\left(x_{1}-\right.$ $\left.x_{2}\right)\|+\| \mathbf{d}\left(s_{2}-s_{1}\right) \|_{\mathbb{A}}$. Due to strict monotonicity of the dilation $\mathbf{d}$ we have $\left\|\mathbf{d}\left(-s_{1}\right)\right\|_{\mathbb{A}} \leq e^{-\beta s_{1}}$ and $\left\|\mathbf{d}\left(s_{2}-s_{1}\right)\right\|_{\mathbb{A}} \leq e^{\beta\left(s_{2}-s_{1}\right)}$ if $0 \leq s_{2} \leq s_{1}$. For $1 \leq$ $\left\|x_{2}\right\|_{\mathbf{d}} \leq\left\|x_{1}\right\|_{\mathbf{d}}$ we have $0 \leq s_{2}<s_{1}$ and, consequently, $1 \leq e^{-\bar{\beta} s_{1}}\left\|x_{1}-x_{2}\right\|+$ $e^{\beta s_{2}-\beta s_{1}}$ or equivalently, $e^{\beta s_{1}}-e^{\beta s_{2}}=\left\|x_{1}\right\|_{\mathbf{d}}^{\beta}-\left\|x_{2}\right\|_{\mathbf{d}}^{\beta} \leq\left\|x_{1}-x_{2}\right\|$. Lipschitz continuity follows from the proven inequality, the identity $\|\mathbf{d}(s) x\|_{\mathbf{d}}=e^{s}\|x\|_{\mathbf{d}}$ and monotonicity of the dilation. The existence of the unique function $s$ : $\mathbb{R}^{n} \rightarrow \mathbb{R}$ such that $\|\mathbf{d}(-s(x)) x\|=1$ has been proven in Theorem 1. Since the dilation is strictly monotone then $\frac{d}{d s}\|\mathbf{d}(-s) x\|<0$ on $S$ (and, on $\mathbb{R}^{n} \backslash\{\mathbf{0}\}$ ) for all $s \in \mathbb{R}$ (see, Theorem 1). Since the norm $\|\cdot\|$ is smooth outside the origin then $\frac{d}{d s}\|\mathbf{d}(-s) x\|=-\left.\frac{\partial\|z\|}{\partial z}\right|_{z=\mathbf{d}(-s) x} G_{\mathbf{d}} \mathbf{d}(-s) x$. Taking into account $\frac{\partial}{\partial x}\|x\|_{\mathbf{d}}=$ $\left.e^{s} \frac{\partial s}{\partial x}\right|_{s=\ln \|x\|_{\mathbf{d}}}$ the formula (7) is derived by means of Implicit Function Theorem [39] implying $\frac{\partial s}{\partial x}=-\frac{\frac{\partial\|\mathbf{d}(-s) x\|}{\partial x}}{\frac{\partial\|\mathbf{d}(-s) x\|}{\partial s}}$ for the function $s: \mathbb{R}^{n} \rightarrow \mathbb{R}$ implicitly defined by the equality $\|\mathbf{d}(-s) x\|=1$.

\subsection{Generalized Homogeneous Functions and Vectors Fields}

Vector fields, which are symmetric (homogeneous) in a certain sense with respect to dilation $\mathbf{d}$, have a lot of properties useful for control design and state estimation of both linear and nonlinear plants as well as for analysis of convergence rates [5], [25], [29], [14].

Definition 5 A vector field $f: \mathbb{R}^{n} \rightarrow \mathbb{R}^{n}$ (resp. a function $h: \mathbb{R}^{n} \rightarrow \mathbb{R}$ ) is said to be $\boldsymbol{d}$-homogeneous of degree $\nu \in \mathbb{R}$ if

$$
\begin{array}{ccc}
f(\boldsymbol{d}(s) x)=e^{\nu s} \boldsymbol{d}(s) f(x), & \forall x \in \mathbb{R}^{n} \backslash\{\mathbf{0}\}, & \forall s \in \mathbb{R} . \\
\left(\text { resp. } h(\boldsymbol{d}(s) x)=e^{\nu s} h(x),\right. & \forall x \in \mathbb{R}^{n} \backslash\{\mathbf{0}\}, & \forall s \in \mathbb{R} .)
\end{array}
$$

Example 1 Let us consider the dilation

$$
\boldsymbol{d}(s)=e^{s}\left(\begin{array}{ccc}
1 & 0 & 0 \\
0 & \cos (s) & \sin (s) \\
0 & -\sin (s) & \cos (s)
\end{array}\right)
$$


that is strictly monotone with respect to the Euclidean norm $\|x\|=\sqrt{x^{T} x}$ and $G_{\boldsymbol{d}}=\left(\begin{array}{ccc}1 & 0 & 0 \\ 0 & 1 & 1 \\ 0 & -1 & 1\end{array}\right)$. The vector field $f: \mathbb{R}^{3} \rightarrow \mathbb{R}^{3}$ defined as

$$
f(x)=\left(\begin{array}{c}
x_{2}^{2}+x_{3}^{2} \\
x_{1}^{2}\left(\cos \left(\ln \left|x_{1}\right|\right)+\sin \left(\ln \left|x_{1}\right|\right)\right) \\
x_{1}^{2}\left(\cos \left(\ln \left|x_{1}\right|\right)-\sin \left(\ln \left|x_{1}\right|\right)\right)
\end{array}\right)
$$

satisfies (8) with $\nu=1$ and the function $h: \mathbb{R}^{3} \rightarrow \mathbb{R}$ given by $h=x_{1}^{3}+\left(x_{2}^{2}+x_{3}^{2}\right)^{\frac{3}{2}}$ satisfies (9) with $\nu=3$, respectively.

Example 2 (Linear homogeneous vector field of arbitrary degree) The vector field may have different degrees of homogeneity dependently of the dilation group. Indeed, the linear vector field $f: \mathbb{R}^{n} \rightarrow \mathbb{R}^{n}, f(x)=A x, x \in \mathbb{R}^{n}$ defined by a chain of integrators $A=\left(\begin{array}{cc}0 & I_{n-1} \\ 0 & 0\end{array}\right) \in \mathbb{R}^{n \times n}$ is $\boldsymbol{d}$-homogeneous of degree $\mu \in[-1,1]$ with $\boldsymbol{d}(s)=\operatorname{diag}\left\{e^{(n+(i-1) \mu) s}\right\}_{i=1}^{n}$.

Remark 1 In the general case, if $G_{d} \in \mathbb{R}^{n \times n}$ is a generator of the dilation, i.e. $\boldsymbol{d}(s)=e^{G_{d} s}, s \in \mathbb{R}$, then a linear map $f: \mathbb{R}^{n} \rightarrow \mathbb{R}^{n}, f(x)=A x, x \in \mathbb{R}^{n}$, $A \in \mathbb{R}^{n \times n}$ is $\boldsymbol{d}$-homogeneous of degree $\nu \in \mathbb{R}$ if

$$
A G_{d}=\left(\nu I_{n}+G_{d}\right) A .
$$

Indeed, in our case the identity (8) becomes $A e^{G_{d} s}=e^{\left(\nu I+G_{d}\right) s} A$. Since $e^{G_{d} s}=$ $\sum_{i=0}^{+\infty} \frac{G_{d}^{i} s^{i}}{i !}$ then the homogeneity identity, obviously, holds provided that (10) is fulfilled.

Let $\mathbb{F}_{\mathbf{d}}\left(\mathbb{R}^{n}\right)$ (resp. $\mathbb{H}_{\mathbf{d}}\left(\mathbb{R}^{n}\right)$ ) be the set of vector fields $\mathbb{R}^{n} \rightarrow \mathbb{R}^{n}$ (resp. functions $\mathbb{R}^{n} \rightarrow \mathbb{R}$ ) satisfying the identity (8) (resp. (9)), which are continuous on $\mathbb{R}^{n} \backslash\{\mathbf{0}\}$. Let $\operatorname{deg}_{\mathbb{F}_{\mathbf{d}}}(f)$ (resp. $\operatorname{deg}_{\mathbb{H}_{\mathbf{d}}}(h)$ ) denote the homogeneity degree of $f \in \mathbb{F}_{\mathbf{d}}\left(\mathbb{R}^{n}\right)\left(\right.$ resp. $\left.h \in \mathbb{H}_{\mathbf{d}}\left(\mathbb{R}^{n}\right)\right)$.

Remark 2 Note that if $n=1$ then the generator of the dilation $G_{d} \in \mathbb{R}_{+}$is a positive scalar and $\boldsymbol{d}(s)=e^{s G_{d}} \in \mathbb{R}_{+}$for any $s \in \mathbb{R}$. Hence, obviously, $\mathbb{F}_{\boldsymbol{d}}(\mathbb{R})=$ $\mathbb{H}_{\boldsymbol{d}}(\mathbb{R})$ but $\operatorname{deg}_{\mathbb{F}_{d}}(\cdot)+G_{\boldsymbol{d}}=\operatorname{deg}_{\mathbb{H}_{d}}(\cdot)$ if $n=1$. In other words, homogeneous vector fields/function $\mathbb{R} \rightarrow \mathbb{R}$ belongs to both sets $\mathbb{F}_{\boldsymbol{d}}(\mathbb{R})$ and $\mathbb{H}_{\boldsymbol{d}}(\mathbb{R})$, but its degree can be defined differently (i.e. according to (8) or (9)) dependently of the context (e.g. if $f$ defines the right-hand side of ODE then the formula (8) is utilized).

The homogeneity allows local properties (e.g. smoothness) of vector fields (functions) to be extended globally [2], [3]. Below we present two useful corollaries to Definition 5.

Corollary 1 The vector field $f \in \mathbb{F}_{\boldsymbol{d}}\left(\mathbb{R}^{n}\right)$ is Lipschitz continuous (smooth) on $\mathbb{R}^{n} \backslash\{\mathbf{0}\}$ if and only if it satisfies Lipschitz condition (it is smooth) on the unit sphere $S$ provided that $\boldsymbol{d}$ is strictly monotone on $\mathbb{R}^{n}$ equipped with a (smooth on $\left.\mathbb{R}^{n} \backslash\{\mathbf{0}\}\right)$ norm $\|\cdot\|$. 
Proof. The necessity is straightforward since any locally Lipschitz function on a compact set satisfies Lipschitz condition on it. Let us prove sufficiency. Let $x_{i} \in \mathbb{R}^{n} \backslash\{\mathbf{0}\}, i=1,2$ then $x_{i}=\mathbf{d}\left(\ln \left\|x_{i}\right\|_{\mathbf{d}}\right) z_{i}$ for some $z_{i} \in S$,

$$
\begin{gathered}
f\left(x_{1}\right)-f\left(x_{2}\right)=f\left(\mathbf{d}\left(\ln \left\|x_{1}\right\|_{\mathbf{d}}\right) z_{1}\right)-f\left(\mathbf{d}\left(\ln \left\|x_{2}\right\|_{\mathbf{d}}\right) z_{2}\right) \\
=\left\|x_{1}\right\|_{\mathbf{d}}^{\nu} \mathbf{d}\left(\ln \left\|x_{1}\right\|_{\mathbf{d}}\right) f\left(z_{1}\right)-\left\|x_{2}\right\|_{\mathbf{d}}^{\nu} \mathbf{d}\left(\ln \left\|x_{2}\right\|_{\mathbf{d}}\right) f\left(z_{2}\right) \\
=\left\|x_{1}\right\|_{\mathbf{d}}^{\nu} \mathbf{d}\left(\ln \left\|x_{1}\right\|_{\mathbf{d}}\right)\left(f\left(z_{1}\right)-f\left(z_{2}\right)\right)+\left(\left\|x_{1}\right\|_{\mathbf{d}}^{\nu} \mathbf{d}\left(\ln \left\|x_{1}\right\|_{\mathbf{d}}\right)\right. \\
\left.-\left\|x_{2}\right\|^{\nu} \mathbf{d}\left(\ln \left\|x_{1}\right\|_{\mathbf{d}}\right)\right) f\left(z_{2}\right)+\left\|x_{2}\right\|^{\nu}\left(\mathbf{d}\left(\ln \left\|x_{1}\right\|_{\mathbf{d}}\right)-\mathbf{d}\left(\ln \left\|x_{2}\right\|_{\mathbf{d}}\right)\right) f\left(z_{2}\right) .
\end{gathered}
$$

If $L>0$ is a Lipschitz constant of $f$ on $S$ then

$$
\begin{gathered}
\left\|f\left(x_{1}\right)-f\left(x_{2}\right)\right\| \leq L\left\|x_{1}\right\|_{\mathbf{d}}^{\nu} \mathbf{d}\left(\ln \left\|x_{1}\right\|_{\mathbf{d}}\right)\left\|z_{1}-z_{2}\right\| \\
+\left\|\mathbf{d}\left(\ln \left\|x_{1}\right\|_{\mathbf{d}}\right) f\left(z_{2}\right)\right\|\left(\left\|x_{1}\right\|_{\mathbf{d}}^{\nu}-\left\|x_{2}\right\|_{\mathbf{d}}^{\nu}\right) \\
+\left\|f\left(z_{2}\right)\right\|\left\|x_{2}\right\|^{\nu}\left\|\mathbf{d}\left(\ln \left\|x_{1}\right\|_{\mathbf{d}}\right)-\mathbf{d}\left(\ln \left\|x_{2}\right\|_{\mathbf{d}}\right)\right\|_{\mathbb{A}} .
\end{gathered}
$$

Since $\mathbf{d}\left(s_{1}\right)-\mathbf{d}\left(s_{2}\right)=G_{\mathbf{d}} \int_{s_{2}}^{s_{1}} \mathbf{d}(s) d s$ and the function $\|\mathbf{d}(\cdot)\|_{\mathbb{A}}$ is strictly monotone increasing then $\left.\| \mathbf{d}\left(\ln \left\|x_{1}\right\|_{\mathbf{d}}\right)-\mathbf{d}\left(\ln \left\|x_{2}\right\|_{\mathbf{d}}\right)\right)\left\|_{\mathbb{A}} \leq M\right\| G_{\mathbf{d}}\|\mid \ln \| x_{1}\left\|_{\mathbf{d}}-\ln \right\| x_{2} \|_{\mathbf{d}}{ }_{\text {, }}$, where $M=\max \left\{\left\|\mathbf{d}\left(\ln \left\|x_{1}\right\|_{\mathbf{d}}\right)\right\|_{\mathbb{A}},\left\|\mathbf{d}\left(\ln \left\|x_{2}\right\|_{\mathbf{d}}\right)\right\|_{\mathbb{A}}\right\}$. Since the homogeneous norm is Lipschitz continuous (see Proposition 1) on $\mathbb{R}^{n} \backslash\{\mathbf{0}\}$ but power and logarithm functions are Lipschitz continuous outside zero then $f$ is Lipschitz continuous outside the origin. (Differentiability of homogeneous vector field (function) $f$ on $\mathbb{R}^{n} \backslash\{\boldsymbol{0}\}$ can be proven by means of the formula (7), the identity $\frac{d}{d s} \mathbf{d}(s)=G_{\mathbf{d}} \mathbf{d}(s)$ and the representation $f(x)=\|x\|_{\mathbf{d}}^{\nu} \mathbf{d}\left(\ln \|x\|_{\mathbf{d}}\right) f(z)$ with $z=\mathbf{d}(-\ln \|x\|) x \in S$.)

If a function (or a vector field) is smooth then homogeneity is inherited by its derivatives in a certain way.

Corollary 2 If $h \in \mathbb{H}_{\boldsymbol{d}}\left(\mathbb{R}^{n}\right) \cap C^{1}\left(\mathbb{R}^{n} \backslash\{\mathbf{0}\}, \mathbb{R}\right)$ then

$$
\begin{gathered}
e^{\operatorname{deg}_{\mathbb{H}_{d}}(h) s \frac{\partial h(x)}{\partial x}}=\left.\frac{\partial h(z)}{\partial z}\right|_{z=d(s) x} \boldsymbol{d}(s), \\
\frac{\partial h(x)}{\partial x} G_{\boldsymbol{d}} x=\operatorname{deg}_{\mathbb{H}_{\boldsymbol{d}}}(h) h(x),
\end{gathered}
$$

for $x \in \mathbb{R}^{n} \backslash\{\mathbf{0}\}$ and $s \in \mathbb{R}$.

Proof. The formula (11) can be obtained using the definition of the (Frechét) derivative, which coincides with $\frac{\partial h}{\partial x}$ if $h$ is smooth. Namely,

$$
\lim _{\|\Delta\| \rightarrow 0} \frac{\left|h(x+\Delta)-h(x)-\frac{\partial h(x)}{\partial x} \Delta\right|}{\|\Delta\|}=0 \text { and } \lim _{\|\Delta\| \rightarrow 0} \frac{\left|h(\mathbf{d}(s) x+\Delta)-h(\mathbf{d}(s) x)-\frac{\partial h(z)}{\partial z}\right|_{z=\mathbf{d}(s) x} \Delta \mid}{\|\Delta\|}=0,
$$

where $\Delta \in \mathbb{R}^{n}$. Since $h \in \mathbb{H}_{\mathbf{d}}$ then

$$
\frac{\left|h(\mathbf{d}(s) x+\Delta)-h(\mathbf{d}(s) x)-\frac{\partial h(z)}{\partial z}\right|_{z=\mathbf{d}(s) x} \Delta \mid}{\|\Delta\|}=e^{\nu s} \frac{\left|h(x+\tilde{\Delta})-h(x)-e^{-\nu s} \frac{\partial h(z)}{\partial z}\right|_{z=\mathbf{d}(s) x} \mathbf{d}(s) \tilde{\Delta} \mid}{\|\mathbf{d}(s) \tilde{\Delta}\|}
$$




$$
\leq \frac{e^{\nu s}}{[\mathbf{d}(s)\rfloor_{\mathbb{A}}} \times \frac{\left|h(x+\tilde{\Delta})-h(x)-e^{-\nu s} \frac{\partial h(z)}{\partial z}\right|_{z=\mathbf{d}(s) x} \mathbf{d}(s) \tilde{\Delta} \mid}{\|\tilde{\Delta}\|},
$$

where $\nu=\operatorname{deg}_{\mathbb{H}_{\mathbf{d}}}(h)$ and $\tilde{\Delta}=\mathbf{d}(-s) \Delta$ such that $\|\tilde{\Delta}\| \rightarrow 0$ implies $\|\Delta\| \rightarrow 0$. Therefore the identity (11) holds.

To prove (12) let us consider the canonical homogeneous norm $\|\cdot\|_{\mathbf{d}}$ induced by a weighted Euclidean norm $\|x\|=\sqrt{x^{\top} P x}, x \in \mathbb{R}^{n} \backslash\{\mathbf{0}\}, P=P^{\top}>0$ satisfying (5). In this case, due to (7) we have $\frac{\partial\|x\|_{\mathbf{d}}}{\partial x} x>0$ if $x \neq 0$. From $h(x)=\|x\|_{\mathbf{d}}^{\nu} h\left(\mathbf{d}\left(-\ln \|x\|_{\mathbf{d}}\right) x\right)$ we derive

$$
\begin{gathered}
\frac{\partial h(x)}{\partial x}=\nu h\left(\mathbf{d}\left(-\ln \|x\|_{\mathbf{d}}\right) x\right)\|x\|_{\mathbf{d}}^{\nu-1} \frac{\partial\|x\|_{\mathbf{d}}}{\partial x}+\|x\|_{\mathbf{d}}^{\nu} \frac{\partial h\left(\mathbf{d}\left(-\ln \|x\|_{\mathbf{d}}\right) x\right)}{\partial x} \\
\left.=\nu h\left(\mathbf{d}\left(-\ln \|x\|_{\mathbf{d}}\right) x\right)\|x\|_{\mathbf{d}}^{\nu-1} \frac{\partial\|x\|_{\mathbf{d}}}{\partial x}+\left.\|x\|_{\mathbf{d}}^{\nu} \frac{\partial h(z)}{\partial z}\right|_{z=\mathbf{d}(-\ln \| x} \|_{\mathbf{d}}\right) x \\
=\nu h\left(\mathbf{d}\left(-\ln \|x\|_{\mathbf{d}}\right) x\right)\|x\|_{\mathbf{d}}^{\nu-1} \frac{\partial\|x\|_{\mathbf{d}}}{\partial x}+\left.\|x\|_{\mathbf{d}}^{\nu} \frac{\partial h(z)}{\partial z}\right|_{z=\mathbf{d}\left(-\ln \|x\|_{\mathbf{d}}\right) x} \\
\times\left(\mathbf{d}\left(-\ln \|x\|_{\mathbf{d}}\right)-\frac{G_{\mathbf{d}} \mathbf{d}\left(-\ln \|x\|_{\mathbf{d}}\right) x}{\|x\|_{\mathbf{d}}} \frac{\partial\|x\|_{\mathbf{d}}}{\partial x}\right) .
\end{gathered}
$$

Therefore, for $\|x\|=1$ we have $\frac{\partial h(x)}{\partial x} G_{\mathbf{d}} x \frac{\partial\|x\|_{\mathbf{d}}}{\partial x}=\nu h(x) \frac{\partial\|x\|_{\mathbf{d}}}{\partial x}$. Hence, multiplying by $x$ we obtain that (12) holds for $\|x\|=1$. Since $\|\cdot\|_{\text {new }}=\gamma\|\cdot\|$ with $\gamma>0$ is again a weighted Euclidean norm in $\mathbb{R}^{n}$ satisfying (5) then the obtained identity holds on $\mathbb{R}^{n} \backslash\{\mathbf{0}\}$.

\section{Quadratic Stability of Homogeneous Systems}

Homogeneity may simplify the analysis of differential equations, e.g. to prove existence and uniqueness of solution on $\mathbb{R}^{n} \backslash\{\mathbf{0}\}$ it is sufficient to prove that the right hand side is Lipschitz continuous (or differentiable) on a sphere (see, Corollary 2. The most important property of $\mathbf{d}$-homogeneous systems is scalability of the solutions [2], [4], [13], [29], [30], [20].

Theorem 3 If $\varphi_{\xi_{0}}:[0, T) \rightarrow \mathbb{R}^{n}$ is a solution to

$$
\dot{\xi}=f(\xi), \quad f \in \mathbb{F}_{\boldsymbol{d}}\left(\mathbb{R}^{n}\right)
$$

with the initial condition $\xi(0)=\xi_{0} \in \mathbb{R}^{n}$ then $\varphi_{d(s) \xi_{0}}:\left[0, e^{-\nu s} T\right) \rightarrow \mathbb{R}^{n}$ defined as $\varphi_{\boldsymbol{d}(s) \xi_{0}}(t):=\boldsymbol{d}(s) \varphi_{\xi_{0}}\left(t e^{\nu s}\right)$ with $s \in \mathbb{R}$ is a solution to (13) with the initial condition $\xi(0)=\boldsymbol{d}(s) \xi_{0}$, where $\nu=\operatorname{deg}_{\mathbb{F}_{d}}(f)$.

Proof. The scheme of the proof is standard (see [2]) for any type of homogeneity. Since $\frac{d}{d t} \varphi_{\xi_{0}}(t)=f\left(\varphi_{\xi_{0}}(t)\right)$ then $\mathbf{d}(s) \frac{d}{d t} \varphi_{\xi_{0}}(t)=\frac{d}{d t} \mathbf{d}(s) \varphi_{\xi_{0}}(t)=$ $\mathbf{d}(s) f\left(\varphi_{\xi_{0}}(t)\right)=e^{-\nu s} f\left(\mathbf{d}(s) \varphi_{\xi_{0}}(t)\right)$. Making the change of time $t=e^{\nu s} t^{\text {new }}$ we complete the proof.

This theorem has a lot of corollaries, which are very useful for qualitative analysis of homogeneous systems. For example, local stability always implies the global one or the existence of strictly invariant (in forward time) compact set implies asymptotic stability [4], [13], [2], etc. Moreover, using the classical result [2], [13] about existence of homogeneous Lyapunov function for asymptotically stable homogeneous system we can prove topological equivalence of such system to a quadratically stable one. 
Theorem 4 The next five claims are equivalent

1) The origin of the system (13) is asymptotically stable.

2) There exists a Lyapunov function $V \in \mathbb{H}_{\boldsymbol{d}}\left(\mathbb{R}^{n}\right) \cap C^{\infty}\left(\mathbb{R}^{n}\right)$;

3) The origin of the system

$$
\dot{z}=\|z\|^{1+\operatorname{deg}_{\mathbb{F}_{d}}(f)}\left(\frac{\left(I_{n}-G_{d}\right) z^{\top} z P}{z^{\top} P G_{d} z}+I_{n}\right) f\left(\frac{z}{\|z\|}\right)
$$

is asymptotically stable, where $\|z\|=\sqrt{z^{\top} P z}$ with $P$ satisfying (5).

4) For any matrix $P \in \mathbb{R}^{n \times n}$ satisfying (5) there exists $\Psi \in \mathbb{F}_{\boldsymbol{d}}\left(\mathbb{R}^{n}\right) \cap$ $C^{\infty}\left(\mathbb{R}^{n} \backslash\{\mathbf{0}\}\right), \operatorname{deg}_{\mathbb{F}_{d}}(\Psi)=0$ such that $\Psi$ is diffemorphism on $\mathbb{R}^{n} \backslash\{\mathbf{0}\}$, homeomorphism on $\mathbb{R}^{n}, \Psi(\mathbf{0})=\mathbf{0}$ and

$$
\frac{\partial\left(\Psi^{\top}(\xi) P \Psi(\xi)\right)}{\partial \xi} f(\xi)<0 \quad \text { if } \Psi^{\top}(\xi) P \Psi(\xi)=1 .
$$

Moreover, $\|\Psi\|_{d} \in \mathbb{H}_{\boldsymbol{d}}\left(\mathbb{R}^{n}\right) \cap C^{\infty}\left(\mathbb{R}^{n} \backslash\{\mathbf{0}\}\right)$ is Lyapunov function to the system (13), where $\|\cdot\|_{d}$ is the canonical homogeneous norm induced by $\|\xi\|=\sqrt{\xi^{\top} P \xi}$.

5) For any matrix $P \in \mathbb{R}^{n \times n}$ satisfying (5) there exists a map

$$
\Xi \in C^{\infty}\left(\mathbb{R}^{n} \backslash\{\mathbf{0}\}, \mathbb{R}^{n \times n}\right)
$$

such that

$$
\operatorname{det}(\Xi(z)) \neq 0, \quad \frac{\partial \Xi(z)}{\partial z_{i}} z=0, \quad \Xi\left(e^{s} z\right)=\Xi(z)
$$

for $z=\left(z_{1}, \ldots, z_{n}\right)^{\top} \in \mathbb{R}^{n} \backslash\{\mathbf{0}\}, s \in \mathbb{R}, i=1, \ldots, n$ and

$$
z^{\top} \Xi^{\top}(z) P \Xi(z)\left(\frac{\left(I_{n}-G_{d}\right) z z^{\top} P}{z^{\top} P G_{d} z}+I_{n}\right) f\left(\frac{z}{\sqrt{z^{\top} P z}}\right)<0 .
$$

Proof. Without loss of generality we may assume that $f$ is continuous at zero and $f(\mathbf{0})=\mathbf{0}$, since asymptotic stability of (14) is equivalent to asymptotic stability of the system $\dot{\xi}=\tilde{f}(\xi):=\|\xi\|_{\mathbf{d}}^{-\operatorname{deg}_{\mathbb{F}_{\mathbf{d}}}(f)} f(\xi)$, which is always continuous at the origin.

1) $\Leftrightarrow$ 2) We use the scheme developed in [13]. The Converse Lyapunov Theorem (see, e.g. [40]) implies that there exists a smooth Lyapunov function $V: \mathbb{R}^{n} \rightarrow \mathbb{R}_{+}$. Let the smooth function $a: \mathbb{R} \rightarrow \mathbb{R}_{+}$be defined as $a(\rho)=e^{\frac{1}{1-\rho}}$ if $\rho>0$ and $a(\rho)=0$ if $\rho \leq 1$. Obviously, $a^{\prime}(\rho)>0$ if $\rho>1$. Then the function $V_{h}: \mathbb{R}^{n} \rightarrow \mathbb{R}_{+}$defined as $V_{h}(\xi)=\int_{-\infty}^{+\infty} e^{-s} a(V(\mathbf{d}(s) \xi)) d s$ is d-homogeneous Lyapunov function to the system (13). Indeed, it is well-defined (due to cut-off function $a$ ), smooth, positive definite and radially unbounded. Finally, $V_{h}$ is d-homogeneous $V_{h}(\mathbf{d}(q) \xi)=\int_{-\infty}^{+\infty} e^{-s} a(V(\mathbf{d}(-s+q) \xi)) d s=e^{q} V_{h}(\xi)$ and

$$
\dot{V}_{h}(\xi)=\left.\int_{-\infty}^{+\infty} e^{-s} a^{\prime}(V(\mathbf{d}(-s) \xi)) \frac{\partial V(z)}{\partial z}\right|_{z=\mathbf{d}(s) \xi} \mathbf{d}(s) f(\xi) d s<0
$$


since $f \in \mathbb{F}\left(\mathbb{R}^{n}\right):\left.\frac{\partial V(z)}{\partial z}\right|_{z=\mathbf{d}(s) \xi} ^{\mathbf{d}}(s) f(\xi)=\left.\frac{1}{e^{\nu s}} \frac{\partial V(z)}{\partial z} f(z)\right|_{z=\mathbf{d}(s) \xi}<0$.

1) $\Leftrightarrow 3$ ) Since $P$ satisfies (5) then the dilation $\mathbf{d}$ is strictly monotone on $\mathbb{R}^{n}$ equipped with the norm $\|\xi\|=\sqrt{\xi^{T} P \xi}$. The change of coordinates $z=\|\xi\|_{\mathbf{d}} \mathbf{d}\left(-\ln \|\xi\|_{\mathbf{d}}\right) \xi$ gives $\|z\|=\|\xi\|_{\mathbf{d}}$ and $\xi=\mathbf{d}(\ln \|z\|) \frac{z}{\|z\|}$,

$$
\begin{array}{r}
\dot{z}=\left(I_{n}-G_{\mathbf{d}}\right) \mathbf{d}\left(-\ln \|\xi\|_{\mathbf{d}}\right) \xi \frac{d\|\xi\|_{\mathbf{d}}}{d \underline{d} t}+\|\xi\|_{\mathbf{d}} \mathbf{d}\left(-\ln \|\xi\|_{\mathbf{d}}\right) f(\xi)= \\
\|\xi\|_{\mathbf{d}}\left(I_{n}-G_{\mathbf{d}}\right) \mathbf{d}\left(-\ln \|\xi\|_{\mathbf{d}}\right) \xi \frac{\xi^{\top} \mathbf{d}^{\top}\left(-\ln \|\xi\|_{\mathbf{d}}\right) P \mathbf{d}\left(-\ln \|\xi\|_{\mathbf{d}}\right) f(\xi)}{\xi^{\top} \mathbf{d}^{\top}\left(-\ln \|\xi\|_{\mathbf{d}}\right) P G_{\mathbf{d}} \mathbf{d}\left(-\ln \|\xi\|_{\mathbf{d}}\right) \xi}+ \\
+\|\xi\|_{\mathbf{d}} \mathbf{d}\left(-\ln \|\xi\|_{\mathbf{d}}\right) f(\xi)= \\
\|\xi\|_{\mathbf{d}}\left(\frac{\left(I-G_{\mathbf{d}}\right) \mathbf{d}\left(-\ln \|\xi\|_{\mathbf{d}}\right) \xi \xi \xi^{\top} \mathbf{d}^{\top}\left(-\ln \|\xi\|_{\mathbf{d}}\right) P}{\xi^{\top} \mathbf{d}^{\top}\left(-\ln \|\xi\|_{\mathbf{d}}\right) P G_{\mathbf{d}} \mathbf{d}\left(-\ln \|\xi\|_{\mathbf{d}}\right) \xi}+I_{n}\right) \mathbf{d}\left(-\ln \|\xi\|_{\mathbf{d}}\right) f(\xi) .
\end{array}
$$

Taking into account $f \in \mathbb{F}_{\mathbf{d}}\left(\mathbb{R}^{n}\right)$ we derive (14).

4) $\Rightarrow$ 2) Since $\Psi(\mathbf{d}(s) \xi)=\mathbf{d}(s) \Psi(\xi)$ then $\Psi(\mathbf{0})=\mathbf{0}$ due to continuity at zero. Note also that $\Psi(\xi) \neq \mathbf{0}$ for $\xi \neq 0$, otherwise (i.e. $\left.\exists \xi^{*} \neq 0: \Psi\left(\xi^{*}\right)=\mathbf{0}\right)$, due to homogeneity we derive that $\Psi(\xi)=\mathbf{0}$ on a smooth curve $\left\{\mathbf{d}(s) \xi^{*}, s \in \mathbb{R}\right\}$, which starts at the origin goes to $\infty$. The latter contradicts the assumption that $\Psi$ is diffeomorphism (continuously differentiable invertible map with continuously differentiable inverse) on $\mathbb{R}^{n} \backslash\{\mathbf{0}\}$. Since $\operatorname{deg}_{\mathbb{F}_{\mathbf{d}}}(\Psi)=0$ then $\|\Psi(\mathbf{d}(s) \xi)\|_{\mathbf{d}}=$ $\|\mathbf{d}(s) \Psi(\xi)\|_{\mathbf{d}}=e^{s}\|\Psi(\xi)\|_{\mathbf{d}}$ and the function $\|\Psi(\cdot)\|_{\mathbf{d}}$ is $\mathbf{d}$-homogeneous of degree 1 , radially unbounded, continuous at the origin and continuously differentiable outside the origin. Due to (7) the inequality $\left.\frac{\partial\|\Psi(\xi)\|}{\partial \xi} f(\xi)\right|_{\|\Psi(\xi)\|=1}<0$ implies $\left.\frac{\partial\|\Psi(\xi)\|_{\mathbf{d}}}{\partial \xi} f(\xi)\right|_{\|\Psi(\xi)\|=1}<0$. Applying homogeneity we derive $\frac{\partial\|\Psi(\xi)\|_{\mathbf{d}}}{\partial \xi} f(\xi)<0$ if $\xi \in \mathbb{R}^{n} \backslash\{\mathbf{0}\}$, i.e. $\|\Psi\|_{\mathbf{d}}$ is a Lyapunov function to the system (13).

2) $\Rightarrow 4$ ) Since the origin of the system (13) is asymptotically stable then there exists a smooth d-homogeneous Lyapunov function $\tilde{V}: \mathbb{R}^{n} \rightarrow \mathbb{R}_{+}$of degree $\mu>0$. The function $V=\tilde{V}^{1 / \mu}$ is also Lyapunov function to (13) that is $\mathbf{d}$-homogeneous of degree 1 , continuous at the origin and smooth outside the origin. Let us consider the map $\Psi: \mathbb{R}^{n} \rightarrow \mathbb{R}^{n}$ defined as

$$
\Psi(\xi)=\mathbf{d}\left(\ln \left(\frac{V(\xi)}{\|\xi\|_{\mathbf{d}}}\right)\right) \xi .
$$

Since $\frac{V(\xi)}{\|\xi\|_{\mathbf{d}}} \in \mathbb{H}_{\mathbf{d}}\left(\mathbb{R}^{n}\right), \operatorname{deg}_{\mathbb{H}_{\mathbf{d}}}\left(\frac{V(\xi)}{\|\xi\|_{\mathbf{d}}}\right)=0$ then $\liminf _{\|\xi\| \rightarrow \mathbf{0}} \frac{V(\xi)}{\|\xi\|_{\mathbf{d}}}=\inf _{\xi \in S} \frac{V(\xi)}{\|\xi\|_{\mathbf{d}}}>$ 0 , so $\Psi$ is continuous at $\mathbf{0}$ and $\Psi(\mathbf{0})=\mathbf{0}$. Obviously, $\Psi(\mathbf{d}(s) \xi)=\mathbf{d}(s) \Psi(\xi)$ and $\|\Psi(\xi)\|_{\mathbf{d}}=V(\xi)$. Using (7) we derive (15). The map $\Psi$ is bijection. The inverse map $\Psi^{-1}: \mathbb{R}^{n} \rightarrow \mathbb{R}^{n}$ is given by

$$
\Psi^{-1}(x)=\mathbf{d}\left(-\ln \left(\frac{V(x)}{\|x\|_{\mathbf{d}}}\right)\right) x,
$$

where $x \in \mathbb{R}^{n}$. Indeed, $\Psi^{-1}(\Psi(\xi))=\xi$ and $\Psi\left(\Psi^{-1}(x)\right)=x$ for all $\xi, x \in \mathbb{R}^{n}$. Since $\Psi$ and $\Psi^{-1}$ are continuous at the origin and smooth outside the origin then $\Psi$ is diffeomorphism on $\mathbb{R}^{n} \backslash\{\mathbf{0}\}$ and homeomorphism on $\mathbb{R}^{n}$.

3) $\Rightarrow$ 5) The system (14) is homogeneous of degree $\operatorname{deg}_{\mathbb{F}_{\mathbf{d}}}(f)$ with respect to the dilation $\mathbf{d}_{0}(s)=e^{s}$ with $G_{\mathbf{d}_{0}}=I_{n}$. Note that $P_{0} G_{\mathbf{d}_{0}}+G_{\mathbf{d}_{0}}^{\top} P_{0}=2 P_{0} \succ$ 
0 holds for an arbitrary symmetric positive definite matrix $P_{0}$. Taking into account $\|z\|_{\mathbf{d}_{0}}=\|z\|=\sqrt{z^{\top} P_{0} z}$ we use the claim 4) to obtain the homogeneous Lyapunov function $V$ defined as $V(z)=\left\|\Psi_{0}(z)\right\|_{\mathbf{d}_{0}}^{2}=\Psi_{0}^{\top}(z) P_{0} \Psi_{0}(z)$ for $z \in \mathbb{R}^{n}$. Since $\Psi_{0}$ is diffeomorphism then $\operatorname{det}\left(\frac{\partial \Psi_{0}(z)}{\partial z}\right) \neq 0$ for $z \in \mathbb{R}^{n} \backslash\{\mathbf{0}\}$. Using (12) we derive $\frac{\partial \Psi_{0}(z)}{\partial z} z=\Psi_{0}(z), \frac{\partial V}{\partial z}=2 z^{\top} \Xi^{\top}(z) P_{0} \Xi(z)$ and $\frac{\partial \Xi(z)}{\partial z_{i}} z=0$, where $\Xi(z)=\frac{\partial \Psi_{0}(z)}{\partial z}$. Finally, $\Xi\left(e^{s} z\right)=\Xi(z)$ for $z \in \mathbb{R}^{n} \backslash\{\mathbf{0}\}$ and $s \in \mathbb{R}$ due to (11) and $\operatorname{deg}_{\mathbb{F}_{\mathbf{d}_{0}}}\left(\Psi_{0}\right)=0$.

5) $\Rightarrow$ 3) Let us consider the function $V(z)=z^{\top} \Xi^{\top}(z) P \Xi(z) z, V \in C\left(\mathbb{R}^{n}, \mathbb{R}\right) \cap$ $C^{\infty}\left(\mathbb{R}^{n} \backslash\{\mathbf{0}\}, \mathbb{R}\right)$. Since $\frac{\partial \Xi(z)}{\partial z_{i}} z=0$ then $\frac{\partial V(z)}{\partial z}=2 z^{\top} \Xi^{\top}(z) P \Xi(z)$ and the condition (16) implies that $V$ is the Lyapunov function to (14).

This theorem proves three important facts:

- Any generalized homogeneous system is diffeomorphic to a standard homogeneous one.

- Any asymptotically stable generalized homogeneous system is diffeomorphic to a quadratically stable one. Indeed, making the change of variables $z=\Psi(\xi)$ we derive $\dot{z}=\tilde{f}(z)$, where $\tilde{f}(z)=$ $\left.\frac{\partial \Psi(\xi)}{\partial \xi} f(\xi)\right|_{\xi=\Psi^{-1}(z)}$, but the criterion (15) implies that $z^{\top} P \dot{z}<0$ if $z^{\top} P z=1$, so the homogeneous norm $\|\cdot\|_{\mathbf{d}}$ is the Lyapunov function to the latter system. Finally, the change of variable $x=$ $\|z\|_{\mathbf{d}} \mathbf{d}\left(-\ln \|z\|_{\mathbf{d}}\right) z$ gives $\|z\|_{\mathbf{d}}=\|x\|$, so the transformed system $\dot{x}=\hat{f}(x)$ is quadratically stable.

- The formula (16) gives a more or less constructive stability criterion. Since $\Xi(z)=\Xi\left(e^{s} z\right)$ for $z \in \mathbb{R}^{n} \backslash\{\mathbf{0}\}, s \in \mathbb{R}$ then the map $\Xi$ is constant along any straight line $\left\{e^{s} z: s \in \mathbb{R}\right\}$ if $z \neq \mathbf{0}$. In addition, the property $\frac{\partial \Xi(z)}{\partial z_{i}} z=0$ motivates the conjecture that a quadratic Lyapunov function ( $\Xi \equiv$ const) always exists for asymptotically stable standard homogeneous system. Such assumption was also studied in [9]. However, in the view of [40, Proposition 5.2] this conjecture seems to be wrong. Therefore, the condition

$$
\begin{gathered}
z^{\top} Q_{0}\left(\frac{\left(I_{n}-G_{\boldsymbol{d}}\right) z z^{\top} P}{z^{\top} P G_{d} z}+I_{n}\right) f\left(\frac{z}{\sqrt{z^{\top} P z}}\right)<0 \text { if } z \neq \mathbf{0}, \\
P G_{\boldsymbol{d}}+G_{\boldsymbol{d}} P>0, \quad P>0, \quad Q_{0}>0
\end{gathered}
$$

derived from (16) is just sufficient stability condition. For $Q_{0}=P$ (or, equivalently, $\Xi=I_{n}$ ) we derive the more simplified stability condition $z^{T} \operatorname{Pf}\left(\frac{z}{\sqrt{z^{\top} P z}}\right)<0$. 
Recall [29, 11, 12], if the standard (or weighted) homogeneous system (13) is asymptotically stable and $\operatorname{deg}_{\mathbb{F}_{\mathbf{d}}}(f)<0$ then it is globally uniformly finite-time stable, i.e there exists a locally bounded function $T: \mathbb{R}^{n} \rightarrow \mathbb{R}$ such that $\varphi_{x_{0}}(t)=0$, $\forall t>T\left(x_{0}\right)$ and $\forall x_{0} \in \mathbb{R}^{n}$. Theorem 4 together with the next proposition give the necessary and sufficient condition of the uniform finite-time stability of the system (13).

Proposition 2 An asymptotically stable $\boldsymbol{d}$-homogeneous system (13) is uniformly finite-time stable if and only if $\operatorname{deg}_{\mathbb{F}_{d}}(f)<0$.

Proof. Recall that in the case of homogeneous system local stability is equivalent to global one. Sufficiency is well known (see, e.g. [7], [29], [20]). Necessity. Suppose the contrary, i.e. $\operatorname{deg}_{\mathbb{F}_{\mathbf{d}}}(f) \geq 0$ and there exists a locally bounded function $T: \mathbb{R}^{n} \backslash\{\boldsymbol{0}\} \rightarrow \mathbb{R}_{+}$such that $\varphi_{\xi_{0}}(t)=0$ for all $t \geq T\left(\xi_{0}\right)$. Uniform finite-time stability of (13) implies (see, [30]) existence of a Lyapunov function such that $T\left(\xi_{0}\right) \leq C V^{\nu}\left(\xi_{0}\right)$ where $\nu>0$ and $C>0$ are some constants. Hence, $T$ is continuous at $\mathbf{0}$ and $T(\mathbf{0})=0$. On the other hand Theorem 3 implies that $T\left(\mathbf{d}(s) \xi_{0}\right)=e^{-\operatorname{deg}_{\mathbb{F}_{\mathbf{d}}}(f) s} T\left(\xi_{0}\right)$ for any $\xi_{0} \in \mathbb{R}^{n}$. If $\operatorname{deg}_{\mathbb{F}_{\mathbf{d}}}(f)>0$ then the latter identity immediately implies contradiction to the local boundedness of the function $T$. If $\operatorname{deg}_{\mathbb{F}_{\mathbf{d}}}(f)=0$ then continuity of $T$ at zero and $T(\mathbf{0})=0$ imply the identity $T\left(\xi_{0}\right)=0, \forall \xi_{0} \in \mathbb{R}^{n}$, that is also impossible.

\subsection{Example: Quadratically Stable High Order Sliding Mode Algorithm}

In this section we demonstrate how the obtained results can be utilized for the so-called high order sliding mode control [12] design. The canonical homogeneous norm is utilized below as an implicit Lyapunov function [41].

Let us consider the system

$$
\begin{gathered}
\dot{\sigma}=A \sigma+B u(\sigma), \quad A=\left(\begin{array}{ccccc}
0 & 1 & 0 & \ldots & 0 \\
0 & 0 & 1 & \ldots & 0 \\
\ldots & \ldots & \ldots & \ldots & \\
0 & 0 & 0 & \ldots & 1 \\
0 & 0 & 0 & \ldots & 0
\end{array}\right), \quad B=\left(\begin{array}{c}
0 \\
0 \\
\ldots \\
0 \\
1
\end{array}\right), \\
u(\sigma)=\sum_{i=1}^{n} \frac{k_{i} \sigma_{i}}{N(\sigma)^{n-i+1}}, \quad \sigma=\left(\sigma_{1}, \sigma_{2}, \ldots, \sigma_{n}\right)^{\top} \in \mathbb{R}^{n},
\end{gathered}
$$

and the weighted dilation

$$
\mathbf{d}(s)=e^{G_{\mathbf{d}} s} \quad \text { with } \quad G_{\mathbf{d}}=\left(\begin{array}{ccccc}
n & 0 & \ldots & 0 & 0 \\
0 & n-1 & \ldots & 0 & 0 \\
\ldots & \ldots & \ldots & \ldots & \\
0 & 0 & \ldots & 2 & 0 \\
0 & 0 & \ldots & 0 & 1
\end{array}\right),
$$


where $N: \mathbb{R}^{n} \rightarrow \mathbb{R}$ is a continuous positive definite function that is $\mathbf{d}$-homogeneous of degree 1, i.e. $N(\sigma)>0$ for $\sigma \neq 0$ and

$$
N(\mathbf{d}(s) \sigma)=e^{s} N(\sigma), \quad \sigma \in \mathbb{R}^{n}, \quad s \in \mathbb{R} .
$$

In view of Definition 4 the function $N$ is simply an arbitrary homogeneous norm. Obviously, the control law can be rewritten in the form

$$
u(\sigma)=K \mathbf{d}(-\ln N(\sigma)) \sigma,
$$

where $K=\left(\begin{array}{lllll}k_{1} & k_{2} & \ldots & k_{n-1} & k_{n}\end{array}\right)^{\top} \in \mathbb{R}^{n}$ is a vector of control parameters.

The considered feedback control function $u: \mathbb{R}^{n} \rightarrow \mathbb{R}$ is $\mathbf{d}$-homogeneous of degree 0 , i.e. $u \in \mathbb{H}_{\mathbf{d}}\left(\mathbb{R}^{n}\right)$ and $\operatorname{deg}_{\mathbb{H}}(u)=0$. Indeed,

$$
\begin{aligned}
& u(\mathbf{d}(s) \sigma)=K \mathbf{d}(-\ln N(\mathbf{d}(s) \sigma)) \mathbf{d}(s) \sigma=K \mathbf{d}\left(-\ln \left(e^{s} N(\sigma)\right)\right) \mathbf{d}(s) \sigma \\
= & K \mathbf{d}(-s-\ln N(\sigma)) \mathbf{d}(s) \sigma=K \mathbf{d}(-\ln N(\sigma)) \mathbf{d}(-s) \mathbf{d}(s) \sigma=u(\sigma),
\end{aligned}
$$

$\sigma \in \mathbb{R}^{n} \backslash\{\mathbf{0}\}, s \in \mathbb{R}$. Therefore, $u$ is a quasi-continuous homogeneous sliding mode control [42]. Indeed, it is homogeneous of degree 0, continuous outside the origin and globally bounded:

$$
\begin{gathered}
\|B u(\sigma)\| \leq\|B K\|_{\mathbb{A}}\|\mathbf{d}(-\ln N(\sigma)) \sigma\| \leq\|B K\|_{\mathbb{A}}\left\|\mathbf{d}\left(-\ln \frac{N(\sigma)}{\|\sigma\|_{\mathbf{d}}}\right)\right\|_{\mathbb{A}}\left\|\mathbf{d}\left(-\ln \|\sigma\|_{\mathbf{d}}\right) \sigma\right\| \\
=\|B K\|_{\mathbb{A}}\left\|\mathbf{d}\left(-\ln \frac{N(\sigma)}{\|\sigma\|_{\mathbf{d}}}\right)\right\|_{\mathbb{A}} \leq C<+\infty, \quad \sigma \in \mathbb{R}^{n}
\end{gathered}
$$

since the function $\chi: \mathbb{R}^{n} \rightarrow \mathbb{R}, \chi(\sigma)=\frac{N(\sigma)}{\|\sigma\|_{\mathrm{d}}}$ is homogeneous of degree 0 , and, consequently, globally bounded. Similarly, for $f(\sigma)=A \sigma+B u(\sigma)$ we derive

$$
\begin{aligned}
f(\mathbf{d}(s) \sigma)=A \mathbf{d}(s) \sigma & +B u(\mathbf{d}(s))=e^{-s} \mathbf{d}(s) A \sigma+B u(\sigma)=e^{-s} \mathbf{d}(s)(A \sigma+B u(\sigma)) \\
& =e^{-s} \mathbf{d}(s) f(\sigma), \quad \sigma \in \mathbb{R}^{n} \backslash\{\mathbf{0}\}, \quad s \in \mathbb{R},
\end{aligned}
$$

i.e. the closed loop system has negative homogeneity degree: $\operatorname{deg}_{\mathbb{F}_{\mathbf{d}}}(f)=-1$.

Let us find some sufficient conditions (to the vector $K$ and to the function $N$ ) allowing the finite-time stability of the closed-loop system. Selecting $Q_{0}=P$ in (17) we derive the following sufficient stability condition

$$
z^{\top} P \tilde{f}(z)<0, \quad \tilde{f}(z)=\frac{1}{\|z\|}(A z+B K \mathbf{d}(-\ln \tilde{N}(z)) z), \quad \text { for } \quad z \in \mathbb{R}^{n} \backslash\{\mathbf{0}\},
$$

where $\tilde{N}(z)=N\left(\frac{z}{\|z\|}\right),\|z\|=\sqrt{z^{\top} P z}$ with $P$ satisfying (5). Since $N$ is a continuous and positive definite function then

$$
0<\min _{\xi \in \mathbb{R}^{n}: \xi^{\top} P \xi=1} N(\xi) \leq \tilde{N}(z) \leq \max _{\xi \in \mathbb{R}^{n}: \xi^{\top} P \xi=1} N(\xi)<+\infty
$$

and there exist numbers $\delta_{\min }$ and $\delta_{\max }$ such that

$$
0<\delta_{\min } \leq \tilde{N}(z) \leq \delta_{\max } .
$$


Obviously, if $N(\sigma)=\|\sigma\|_{\mathbf{d}}$ is the canonical homogeneous norm then $\tilde{N}(z)=$ $1, z \in \mathbb{R}^{n} \backslash\{\mathbf{0}\}$ and the stability condition becomes $z^{T} P(A+B K) z<0, z \in$ $\mathbb{R}^{n} \backslash\{\mathbf{0}\}$, i.e. $K$ can be selected as a gain matrix of a linear stabilizing feedback. Since the pair $\{A, B\}$ is controllable then appropriate $K$ and $P \succ 0$ can always be found (see, e.g. [32]) to guarantee the required inequality, while the parameter $\gamma$ can be selected sufficiently large to fulfill (5). Moreover, Theorem 4 implies that $\|\cdot\|_{\mathbf{d}}$ is the Lyapunov function to the closed-loop system.

Therefore, the usage of the canonical homogeneous norm allows the very simple rule to be applied in order to select the parameters of control. However, $\|\cdot\|_{\mathbf{d}}$ is defined implicitly by means of the equation (6), and for simplicity of practical realization it is worth to know if $\|\cdot\|_{\mathbf{d}}$ can be replaced with some explicitly defined homogeneous norm $N$ (see also [41], [43]).

Proposition 3 Let for some $0<\delta_{\min } \leq \delta_{\max }$ the system of LMIs

$$
\left(\begin{array}{ccc}
A X+X A^{\top}+B Y+Y^{\top} B^{\top}-X & \sqrt{\ln \left(\frac{\delta_{\max }}{\delta_{\min }}\right)} B Y & X \boldsymbol{d}\left(\ln \frac{\delta_{\max }}{\delta_{\min }}\right) \\
\sqrt{\ln \left(\frac{\delta_{\max }}{\delta_{\min }}\right) Y^{T} B^{T}} & -\left(G_{\boldsymbol{d}}^{-1} X+X G_{\boldsymbol{d}}^{-1}\right) & \mathbf{0} \\
\boldsymbol{d}\left(\ln \frac{\delta_{\max }}{\delta_{\min }}\right) X & \mathbf{0} & -X
\end{array}\right) \prec 0, X \succ 0,
$$

be feasible with respect to $X \in \mathbb{R}^{n \times n}, Y \in \mathbb{R}^{1 \times n}$. If a function $N: \mathbb{R}^{n} \backslash\{\mathbf{0}\} \rightarrow \mathbb{R}$ is continuous, positive definite, $N \in \mathbb{H}_{\boldsymbol{d}}\left(\mathbb{R}^{n}\right)$, $\operatorname{deg}_{H_{\boldsymbol{d}}}(N)=1$ and it satisfies the inequalities (21) with $P=X^{-1}$ and $0<\delta_{\min } \leq \delta_{\max }$ then the closed-loop system (18), (19) with

$$
K=Y X^{-1} \boldsymbol{d}\left(\ln \delta_{\max }\right)
$$

is globally uniformly finite-time stable and the canonical homogeneous norm $\|\cdot\|_{\boldsymbol{d}}$ is its Lyapunov function.

Proof. First of all, the LMI (22) implies that $G_{\mathbf{d}}^{-1} X+X G_{\mathbf{d}}^{-1} \succ 0$ or, equivalently, $P G_{\mathbf{d}}^{-1}+G_{\mathbf{d}}^{-1} P \succ 0$ and $G_{\mathbf{d}} P+P G_{\mathbf{d}} \succ 0$. Obviously, if

$$
z^{T} P(A+B K \mathbf{d}(-\ln \delta)) z<0, \quad z \in \mathbb{R}^{n} \backslash\{\mathbf{0}\}, \quad \forall \delta \in\left[\delta_{\min }, 1\right] .
$$

then the stability condition (20) is fulfilled. For an arbitrary fixed $z \in \mathbb{R}^{n} \backslash\{\mathbf{0}\}$ let us consider the smooth function $q:\left[\delta_{\min }, 1\right] \rightarrow \mathbb{R}$ defined as

$$
q(\delta)=z^{T} P(A+B K \mathbf{d}(-\ln \delta)) z
$$

To estimate its derivative $q^{\prime}(\delta)$ we use the classical inequality

$$
2 y^{\top} v \leq y^{\top} y+v^{\top} v
$$

that, obviously, holds for all $y, v \in \mathbb{R}^{n}$ and, in particular, for

$$
y=\left(G_{\mathbf{d}}^{-1} P+P G_{\mathbf{d}}^{-1}\right)^{-\frac{1}{2}} \mathbf{d}\left(-\ln \delta_{\max }\right) K^{\top} B^{\top} z
$$


and

$$
v=\left(G_{\mathbf{d}}^{-1} P+P G_{\mathbf{d}}^{-1}\right)^{\frac{1}{2}} G_{\mathbf{d}} \mathbf{d}\left(-\ln \frac{\delta}{\delta_{\max }}\right) z .
$$

Since $\frac{d}{d s} \mathbf{d}(s)=G_{\mathbf{d}} \mathbf{d}(s)=\mathbf{d}(s) G_{\mathbf{d}}$ for $s \in \mathbb{R}$ (see (4)) then we have

$$
\begin{gathered}
-q^{\prime}(\delta)=\frac{1}{\delta} z^{T} P B K G_{\mathbf{d}} \mathbf{d}(-\ln \delta) z=\frac{1}{\delta} y^{\top} v \leq \\
\frac{z^{\top} P B K \mathbf{d}\left(-\ln \delta_{\max }\right)\left(G_{\mathbf{d}}^{-1} P+P G_{\mathbf{d}}^{-1}\right)^{-1} \mathbf{d}\left(-\ln \delta_{\max }\right) K^{\top} B^{\top} P z+z^{\top} \mathbf{d}\left(-\ln \frac{\delta}{\delta_{\max }}\right)\left(P G_{\mathbf{d}}+G_{\mathbf{d}} P\right) \mathbf{d}\left(-\ln \frac{\delta}{\delta_{\max }}\right) z}{2 \delta} \\
=\frac{\frac{1}{\delta} z^{T} P B K \mathbf{d}\left(-\ln \delta_{\max }\right)\left(G_{\mathbf{d}}^{-1} P+P G_{\mathbf{d}}^{-1}\right)^{-1} \mathbf{d}\left(-\ln \delta_{\max }\right) K^{\top} B^{\top} P z-\frac{d}{d \delta}\left(z^{\top} \mathbf{d}\left(-\ln \frac{\delta}{\delta_{\max }}\right) P \mathbf{d}\left(-\ln \frac{\delta}{\delta_{\max }}\right) z\right)}{2} .
\end{gathered}
$$

Finally, we derive

$$
\begin{gathered}
q(\delta)=q\left(\delta_{\max }\right)-\int_{\delta}^{\delta_{\max }} q^{\prime}(s) d s \leq z^{T} P\left(A+B K \mathbf{d}\left(-\ln \delta_{\max }\right)\right) z \\
+\frac{\ln \left(\frac{\delta_{\max }}{\delta_{\min }}\right) z^{T} P B K \mathbf{d}\left(-\ln \delta_{\max }\right)\left(G_{\mathbf{d}}^{-1} P+P G_{\mathbf{d}}^{-1}\right)^{-1} \mathbf{d}\left(-\ln \delta_{\max }\right) K^{\top} B^{\top} P z-z^{\top} P z+z^{\top} \mathbf{d}\left(-\ln \frac{\delta_{\min }}{\delta_{\max }}\right) P \mathbf{d}\left(-\ln \frac{\delta_{\min }}{\delta_{\max }}\right) z}{2}
\end{gathered}
$$

for all $\delta \in\left[\delta_{\min }, \delta_{\max }\right]$. In other words, the matrix inequality

$$
\begin{aligned}
& P\left(A+B K \mathbf{d}\left(-\ln \delta_{\max }\right)\right)+\left(A+B K \mathbf{d}\left(-\ln \delta_{\max }\right)\right)^{\top} P+\mathbf{d}\left(-\ln \frac{\delta_{\min }}{\delta_{\max }}\right) P \mathbf{d}\left(-\ln \frac{\delta_{\min }}{\delta_{\max }}\right)-P \\
& \quad+\ln \left(\frac{\delta_{\max }}{\delta_{\min }}\right) P B K \mathbf{d}\left(-\ln \delta_{\max }\right)\left(G_{\mathbf{d}}^{-1} P+P G_{\mathbf{d}}^{-1}\right)^{-1} \mathbf{d}\left(-\ln \delta_{\max }\right) K^{\top} B^{\top} P \prec 0
\end{aligned}
$$

implies (20). Using Schur Complement it can be easily shown that the latter matrix inequality is equivalent (22) with $X=P^{-1}$ and $Y=K \mathbf{d}\left(-\ln \delta_{\max }\right) P^{-1}$.

The system of LMIs (22) is always feasible provided that $\delta_{\min }$ and $\delta_{\max }$ are sufficiently close to each other. Indeed, $\ln \left(\delta_{\max } / \delta_{\min }\right) \rightarrow 0$ and $\mathbf{d}\left(\ln \left(\delta_{\max } / \delta_{\min }\right)\right) \rightarrow$ $I_{n}$ as $\delta_{\max } / \delta_{\min } \rightarrow 1$, so the LMI (22) becomes feasible.

A possible explicit selection of $N$ is given by

$N(\sigma)=\left(\phi^{\top}(\sigma) Q \phi(\sigma)\right)^{\frac{1}{2 n}} \quad$ with $\phi(\sigma)=\left(\begin{array}{c}\left|\sigma_{1}\right|^{r_{1}} \operatorname{sign}\left(\sigma_{1}\right) \\ \ldots \\ \left|\sigma_{n}\right|^{r_{n}} \operatorname{sign}\left(\sigma_{n}\right)\end{array}\right), r_{i}=\frac{n}{n-i+1}, \quad i=1,2, \ldots, n$,

where $Q \in \mathbb{R}^{n \times n}$ is a positive definite matrix.

To illustrate the proposition 3 we consider the system (18), (19), (23) for $n=2$. Selecting $\delta_{\min }=1, \delta_{\max }=1.4$ we solve the LMI (22):

$$
P=\left(\begin{array}{ll}
1.1214 & 0.4247 \\
0.4247 & 0.2327
\end{array}\right) \text { and } K=\left(\begin{array}{ll}
-10.8695 & -6.2376
\end{array}\right) \text {. }
$$

One can be checked numerically (e.g. using sufficiently dense grid on the unit sphere $\left\{z \in \mathbb{R}^{n}: z^{\top} P z=1\right\}$ ) that the homogeneous norm (23) with

$$
Q=\left(\begin{array}{ll}
1.9403 & 0.4410 \\
0.4410 & 0.1330
\end{array}\right)
$$




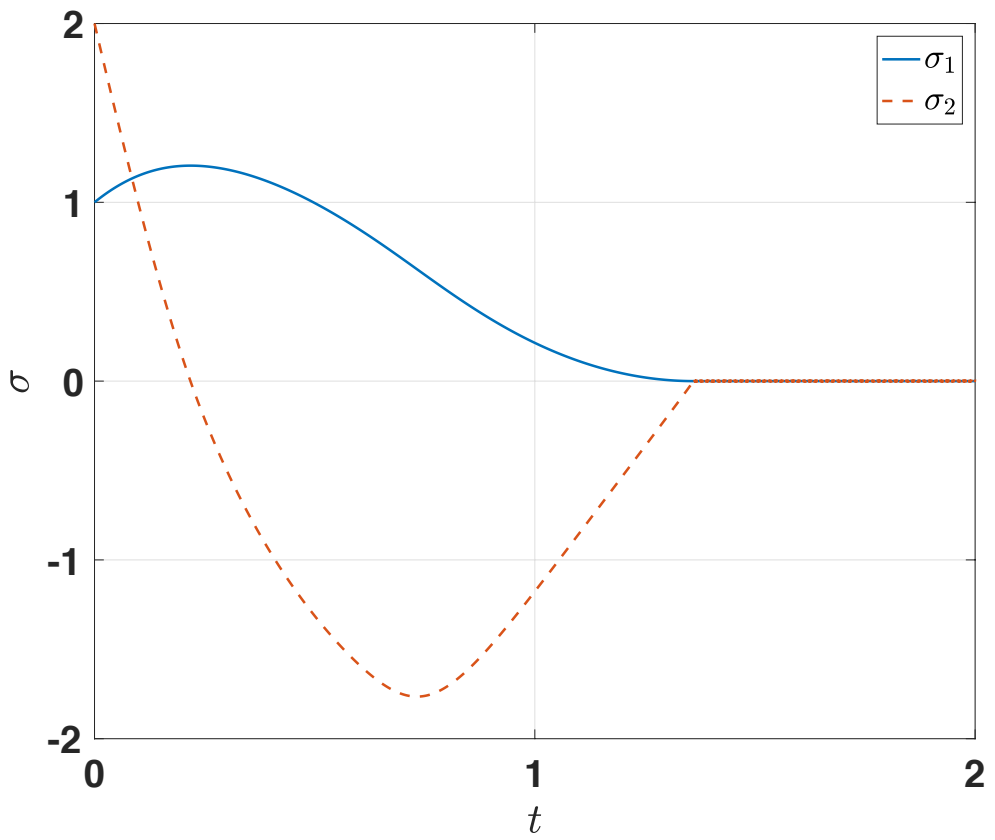

Figure 2: Evolution of the state of the system (18) with the static homogeneous feedback (19), (23).

satisfies $1 \leq N(z) \leq 1.4$ for $z^{\top} P z=1$, i.e. the condition (21) holds. The results of numerical simulation for the considered controller are depicted at Figures 2 and 3 . The system has been discretized by means of the explicit Euler method with the step size 0.002 .

Taking into account Remark 1 the similar control design scheme can be developed for an arbitrary matrix $A$ provided that the pair $\{A, B\}$ is controllable.

\section{Stabilization of Homogeneous Control Systems via Dynamical Feedback in the Disturbance- Free Case}

In this section we study the problem of stabilizability of the homogeneous system (1) in the disturbance free case $(\omega=0)$, i.e.

$$
\dot{\sigma}(t)=g(\sigma(t), u(t)),
$$

by means of the homogeneous dynamical feedback (2):

$$
\dot{u}(t)=k(\sigma(t), u(t)) .
$$

Based on the scheme of universal stabilizing control design given in [44] we derive the following corollary of Theorem 4 . 


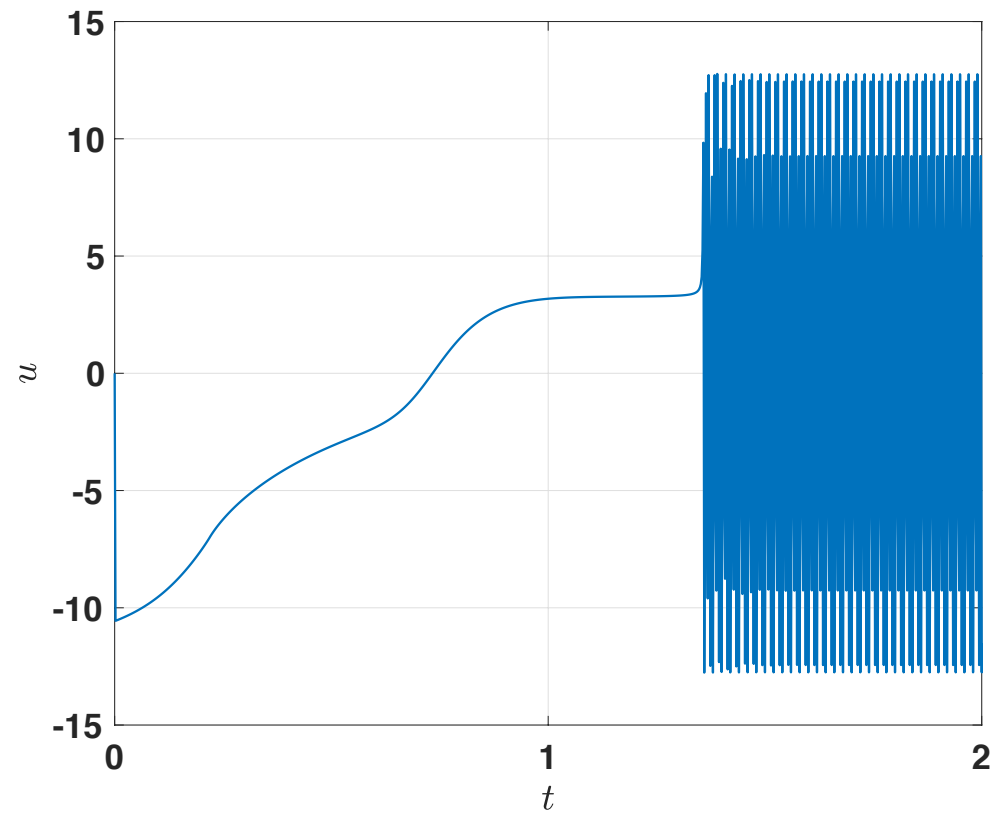

Figure 3: Evolution of the control signal of the system (18) with the static homogeneous feedback (19), (23).

Theorem 5 (On Homogeneous Dynamical Feedback) Let $\boldsymbol{d}_{x}$ and $\boldsymbol{d}_{u}$ be dilations in $\mathbb{R}^{n}$ and $\mathbb{R}^{m}$, respectively,

$$
\boldsymbol{d}:=\left(\begin{array}{cc}
\boldsymbol{d}_{x}(s) & \mathbf{0} \\
\mathbf{0} & \boldsymbol{d}_{u}(s)
\end{array}\right) \text { and } \tilde{f}=\left(\begin{array}{c}
g \\
\mathbf{0}
\end{array}\right) \in \mathbb{F}_{\boldsymbol{d}}\left(\mathbb{R}^{n+m}\right) .
$$

The origin of the system (1) with $\omega=0$ is globally asymptotically stabilizable by means of the homogeneous dynamical feedback (2) with

$$
\left(\begin{array}{c}
g \\
k
\end{array}\right) \in \mathbb{F}_{\boldsymbol{d}}\left(\mathbb{R}^{n+m}\right)
$$

if and only if there exist a number $\gamma \geq 0$, a symmetric matrix $P \in \mathbb{R}^{(n+m) \times(n+m)}$ satisfying (5) and a map $\Xi \in C^{\infty}\left(\mathbb{R}^{n+m} \backslash\{\mathbf{0}\}, \mathbb{R}^{(n+m) \times(n+m)}\right)$ :

$$
\operatorname{det}(\Xi(z)) \neq 0, \frac{\partial \Xi(z)}{\partial z_{i}} z=0, \Xi\left(e^{s} z\right)=\Xi(z)
$$

for $z=\left(z_{1}, . ., z_{n+m}\right) \in \mathbb{R}^{n+m} \backslash\{\mathbf{0}\}, s \in \mathbb{R}, i=1, \ldots, n+m$ such that

$$
a(z)<\gamma \sqrt{b^{\top}(z) b(z)} \quad \text { for } \quad z \in S,
$$

where

$$
\left.\begin{array}{l}
a(z)=z^{\top} W(z) \tilde{f}(z), \quad b^{\top}(z)=z^{\top} W(z)\left(\begin{array}{c}
0 \\
I_{m}
\end{array}\right), \\
W(z)=\Xi^{\top}(z) P \Xi(z)\left(\frac{\left(I_{n+m}-G_{d}\right) z^{\top} z P}{z^{\top} P G_{d} z}+I_{n+m}\right.
\end{array}\right),
$$


and $S$ is the unit sphere in $\mathbb{R}^{n+m}$ with $\|z\|=\sqrt{z^{\top} P z}$. Moreover, the corresponding stabilizing homogeneous feedback law can be designed as follows

$$
k(\xi)=\|\xi\|_{d}^{\operatorname{deg}_{\mathbb{F}_{d}}(\tilde{f})} \boldsymbol{d}_{u}\left(\ln \|\xi\|_{d}\right) k_{0}\left(\boldsymbol{d}\left(-\ln \|\xi\|_{\boldsymbol{d}}\right) \xi\right) \quad \text { with } \quad \xi=\left(\begin{array}{c}
\sigma \\
u
\end{array}\right),
$$

where

$$
k_{0}(\cdot)=\left\{\begin{array}{ccc}
-\frac{a(\cdot)+\sqrt{a^{2}(\cdot)+\left(b^{\top}(\cdot) b(\cdot)\right)^{2}}}{b^{\top}(\cdot) b(\cdot)} & \text { if } & b(\cdot) \neq \mathbf{0} \\
0 & \text { if } b(\cdot)=\mathbf{0}
\end{array}\right.
$$

and $\|\cdot\|_{d}$ is the canonical homogeneous norm induced by $\|\xi\|=\sqrt{\xi^{\top} P \xi}$.

Proof. Sufficiency. Let us show that the function $k_{0}$ is continuous on $S$. Indeed, $k_{0}(z)=\frac{a(z)+\sqrt{a^{2}(z)+\left(b^{\top}(z) b(z)\right)^{2}}}{\sqrt{b^{\top}(z) b(z)}} \frac{b(z)}{\sqrt{b^{\top}(z) b(z)}}$, the first fraction is continuous and equals zero if $b(z)=\mathbf{0}$ (see, [44]), but the norm of the second fraction is globally bounded and continuous for $z \in \mathbb{R}^{n}: b(z) \neq \mathbf{0}$. Let us consider the closedloop system (1), (2), (25): $\dot{\xi}=f(\xi)$, where $f=\left(\begin{array}{l}g \\ k\end{array}\right) \in \mathbb{F}_{\mathbf{d}}\left(\mathbb{R}^{n}\right)$. Under this notation, the stability condition (16) becomes $a(z)+b^{\top}(z) k_{0}(z)<0$. The latter hods for all $z \in S$. Indeed, the inequality (24) implies that $a(z)<0$ if $b(z)=\mathbf{0}$, but for $b(z) \neq \mathbf{0}$ we have $a(z)+b^{\top}(z) k_{0}(z)=a(z)-b^{\top}(z) \frac{a(z)+\sqrt{a^{2}(z)+\left(b^{\top}(z) b(z)\right)^{2}}}{b^{\top}(z) b(z)} b(z)=$ $-\sqrt{a^{2}(z)+\left(b^{\top}(z) b(z)\right)^{2}}<0$.

Necessity. Let us assume that there exists a map $\tilde{k} \in C\left(\mathbb{R}^{n+m} \backslash\{\mathbf{0}\}, \mathbb{R}^{m}\right)$ such that the closed-loop system (1), (2) is globally asymptotically stable and $f=\left(\begin{array}{l}g \\ \tilde{k}\end{array}\right) \in \mathbb{F}_{\mathbf{d}}\left(\mathbb{R}^{n+m}\right)$. Let $\Xi \in C^{\infty}\left(\mathbb{R}^{n+m} \backslash\{\mathbf{0}\}, \mathbb{R}^{(n+m) \times(n+m)}\right)$ be derived according to Theorem 4 such that (16) holds. To show that in this case (24) also holds for some $\gamma \geq 0$, we rewrite the inequality (16) as $a(z)<-b^{\top}(z) \tilde{k}(z)$. Since $\tilde{k} \in C\left(\mathbb{R}^{n+m} \backslash\{\mathbf{0}\}, \mathbb{R}^{m}\right)$ then $\gamma=\max _{z \in S} \sqrt{\tilde{k}^{\top}(z) \tilde{k}(z)}<+\infty$ and using Cauchy-Schwarz inequality we derive

$$
-b^{\top}(z) \tilde{k}(z) \leq \sqrt{\tilde{k}^{\top}(z) \tilde{k}(z)} \sqrt{b^{\top}(z) b(z)} \leq \gamma \sqrt{b^{\top}(z) b(z)} .
$$

For $\Xi(z)=I_{n}$ the inequality (24) gives the following sufficient condition of quadratic stabilizability:

$$
\begin{gathered}
z^{\top} P \tilde{f}\left(\frac{z}{\sqrt{z^{\top} P z}}\right) \leq \gamma \sqrt{z^{\top} P\left(\begin{array}{cc}
0 & 0 \\
0 & I_{m}
\end{array}\right) P z}, \quad z \neq \mathbf{0} \\
P G_{\mathbf{d}}+G_{\mathbf{d}}^{\top} P>0, \quad P>0 .
\end{gathered}
$$

Taking into account Proposition 2 we derive criterion of finite-time stabilizability of the system (1) by means of the feedback (2) in the case $(\omega=0)$.

Example 3 (Quadratic Dynamical Stabilization in a Finite Time) Let us consider the stabilization problem for the system

$$
\dot{\sigma}_{1}=\left|\sigma_{1}\right|^{\frac{1}{3}} u+\sigma_{2}, \quad \dot{\sigma}_{2}=u,
$$


where $\sigma_{1}, \sigma_{2}, u \in \mathbb{R}$. The vector field $\tilde{f}: \mathbb{R}^{3} \rightarrow \mathbb{R}^{3}$ defined by

$$
\tilde{f}(\xi)=\left(\begin{array}{c}
\left|\xi_{1}\right|^{\frac{1}{3}} \xi_{3}+\xi_{2} \\
\xi_{3} \\
0
\end{array}\right),
$$

$\xi=\left(\xi_{1}, \xi_{2}, \xi_{3}\right)^{\top}=\left(\sigma_{1}, \sigma_{2}, u\right)^{\top}$ is $\boldsymbol{d}$-homogeneous of degree -1 with respect to the dilation $\boldsymbol{d}$ in $\mathbb{R}^{3}$ generated by $G_{\boldsymbol{d}}=\operatorname{diag}\{3,2,1\}$. Hence, the sufficient stabilizability condition (27) becomes

$$
z^{\top} P A(z) z<\gamma \sqrt{z^{\top} P z} \sqrt{z^{\top} P e_{3} e_{3}^{\top} P z}
$$

$P>0, P G_{\boldsymbol{d}}+G_{\boldsymbol{d}}^{\top} P>0$, where $z=\left(z_{1}, z_{2}, z_{3}\right)^{\top} \in \mathbb{R}^{3}$ and

$$
A(z)=\left(\begin{array}{ccc}
0 & 1 & \left(\frac{\left|z_{1}\right|}{\sqrt{z^{\top} P z}}\right)^{\frac{1}{3}} \\
0 & 0 & 1 \\
0 & 0 & 0
\end{array}\right) .
$$

Let $X \in \mathbb{R}^{3 \times 3}$ and $\gamma_{0}>0$ satisfy the linear matrix inequalities

$$
X A_{i}+A_{i}^{\top} X-2 \gamma e_{3} e_{3}^{\top}<0, \quad X G_{d}+G_{d} X>0, \quad X>0,
$$

$A_{i}=\left(\begin{array}{lll}0 & 1 & i \\ 0 & 0 & 1 \\ 0 & 0 & 0\end{array}\right), i=0,1, e_{3}=(0,0,1)^{\top} \in \mathbb{R}^{3}$. Since $A(z)=\alpha(z) A_{1}+(1-$ $\alpha(z)) A_{0}$, where $\alpha(z)=\left(\frac{\left|z_{1}\right|}{\sqrt{z^{\top} P z}}\right)^{\frac{1}{3}}$ and $\alpha(z) \in[0,1]$ provided that $p_{11}=1$, then for $P=\tilde{P} /\left(\tilde{p}_{11}\right), \tilde{P}=X^{-1}$ the stabilizability condition (27) holds. So, the considered system can be stabilized in a finite time $\left(\right.$ due to $\left.\operatorname{deg}_{d}(\tilde{f})=-1\right)$ to zero by means of the $\boldsymbol{d}$-homogeneous dynamical feedback

$$
\dot{u}=k_{0}\left(\boldsymbol{d}\left(-\ln \|\xi\|_{\boldsymbol{d}}\right) \xi\right),
$$

where $k_{0}$ is given by (26) with $a(z)=z^{\top} P \tilde{f}(z)$ and $b(z)=p_{13} z_{1}+p_{23} z_{2}+p_{33} z_{3}$. For example, the appropriate matrix $P$ obtained using the above LMIs is

$$
P=\left(\begin{array}{lll}
1.0000 & 0.8930 & 0.8846 \\
0.8930 & 1.8679 & 1.3659 \\
0.8846 & 1.3659 & 1.7523
\end{array}\right)
$$

Remark 3 The canonical homogeneous norm is defined implicitly by the formula (6). Therefore, to implement the designed control law either the canonical homogeneous norm must be calculated numerically on-line (like in [41], [17]) or it must be approximated with some explicitly defined homogeneous norm $N$ (for example, using the formula (23) or the results of [43]). Possibility of such a replacement can studied using robustness properties of the control (see, the next section). The construction of an appropriate approximation $N$ of the canonical homogeneous norm $\|\cdot\|$, in the general case, is a non-trivial theoretical problem needed to be studied in future. 


\section{Robust Stabilization via Dynamical Feedback}

In this section we study the problem of robust stabilizability of the homogeneous system (1):

$$
\dot{\sigma}(t)=g(\sigma(t), u(t))+\omega(t, q(t), u(t)), \quad\|\omega\| \leq \bar{\omega}\left(\left\|\left(\begin{array}{l}
\sigma \\
u
\end{array}\right)\right\|\right),
$$

by means of the homogeneous dynamical feedback (2):

$$
\dot{u}(t)=k(\sigma(t), u(t)) .
$$

Necessary condition is obviously given by Theorem 5 . Sufficient condition asks for an additional restriction to the uncertain term $\omega$.

Corollary 3 Let conditions of Theorem 5 hold and

$$
z^{T} \Xi^{T}(z) P \Xi(z)\left(\frac{\left(I_{n+m}-G_{d}\right) z z^{\top} P}{z^{\top} P G_{d} z}+I_{n+m}\right)\left(f\left(\frac{z}{\|z\|}\right)+\frac{d(-\ln \|z\|) \tilde{\omega}}{\|z\|^{\operatorname{deg}_{\mathbb{d}}(f)}}\right)<0, \quad z \neq \mathbf{0}
$$

where $z=\|\xi\|_{\boldsymbol{d}} \boldsymbol{d}\left(-\ln \|\xi\|_{\boldsymbol{d}}\right) \xi,\|z\|=\sqrt{z^{\top} P z}, \tilde{\omega}=\left(\begin{array}{c}\omega \\ 0\end{array}\right), \xi=\left(\begin{array}{c}\sigma \\ u\end{array}\right) \in \mathbb{R}^{n+m}$. Then the closed-loop system (1), (2) is globally asymptotically stable.

Proof. Making the change of variables $z=\|\xi\|_{\mathbf{d}} \mathbf{d}\left(-\ln \|\xi\|_{\mathbf{d}}\right) \xi$ in the closedloop system (1), (2) we derive (see, Proof of Theorem (4))

$$
\dot{z}=\|z\|\left(\frac{\left(I_{n+m}-G_{\mathbf{d}}\right) z z^{\top} P}{z^{\top} P G_{\mathbf{d}} z}+I_{n+m}\right)\left(\|z\|^{\operatorname{deg}_{\mathbb{F}_{\mathbf{d}}}(f)} f\left(\frac{z}{\|z\|}\right)+\mathbf{d}(-\ln \|z\|) \tilde{\omega}\right) .
$$

Next, using Theorem 4, conditions of Theorem 5 and the inequality (30) we complete the proof.

More constructive (but also more conservative) sufficient condition can be derived under an assumption on quadratic stabilizability of the nominal system.

Corollary 4 Let conditions of Theorem 5 hold for $\Xi=I_{n}$ and

$$
\begin{aligned}
& \sqrt{\tilde{\omega}^{\top} P \tilde{\omega}}<-z^{\top} \operatorname{Pf}\left(\frac{z}{\|z\|}\right)\|z\|^{\alpha+\operatorname{deg}_{\mathbb{F}_{d}}(f)-1} \quad \text { if } \quad 0<\|z\| \leq 1, \\
& \sqrt{\tilde{\omega}^{\top} P \tilde{\omega}}<-z^{\top} \operatorname{Pf}\left(\frac{z}{\|z\|}\right)\|z\|^{\beta+\operatorname{deg}_{\mathbb{F}_{d}}(f)-1} \quad \text { if } \quad\|z\|>1,
\end{aligned}
$$

where $z=\|\xi\|_{d} \boldsymbol{d}\left(-\ln \|\xi\|_{d}\right) \xi,\|z\|=\sqrt{z^{\top} P z}, \tilde{\omega}=\left(\begin{array}{c}\omega \\ 0\end{array}\right), \xi=\left(\begin{array}{l}x \\ u\end{array}\right) \in \mathbb{R}^{n+m}$ and $\alpha, \beta$ are defined in Theorem 2. Then the closed-loop system (1), (2) is globally asymptotically stable.

Proof. For $\Xi=I_{n}$ the inequality (30) is equivalent to

$$
z^{T} P\left(f\left(\frac{z}{\sqrt{z^{\top} P z}}\right)+\frac{\mathbf{d}(-\ln \|z\|) \tilde{w}}{\|z\|^{\operatorname{deg}_{\mathbb{d}}(f)}}\right)<0 .
$$


Using Cauchy-Schwartz inequality we derive

$$
z^{T} P \mathbf{d}\left(-\ln \|z\|_{\mathbf{d}}\right) \tilde{\omega} \leq \sqrt{z^{\top} P z} \sqrt{\tilde{\omega}^{\top} \mathbf{d}^{\top}(-\ln \|z\|) P \mathbf{d}(-\ln \|z\|) \tilde{\omega}} .
$$

and the sufficient condition of robust stability becomes

$$
\sqrt{\tilde{\omega}^{\top} \mathbf{d}^{\top}(-\ln \|z\|) P \mathbf{d}(-\ln \|z\|) \tilde{\omega}}<-\|z\|^{\operatorname{deg}_{\mathbb{P}_{\mathbf{d}}}(f)-1} z^{T} \operatorname{Pf}\left(\frac{z}{\|z\|}\right) .
$$

Denote $\phi(s)=\tilde{\omega}^{\top} \mathbf{d}^{\top}(s) P \mathbf{d}(s) \tilde{\omega}$. Obviously, from Theorem 2 we derive

$$
2 \beta \phi(s) \leq \frac{d}{d s} \phi(s) \leq 2 \alpha \phi(s) \Rightarrow\left\{\begin{array}{l}
\phi(s) \leq e^{2 \alpha s} \phi(0) \text { for } s \geq 0 \\
\phi(s) \leq e^{2 \beta s} \phi(0) \text { for } s \leq 0 .
\end{array}\right.
$$

Hence, denoting $s=-\ln \|z\|$ and using (31) we complete the proof.

Notice that in some particular cases the robust stability condition (32), that is less conservative than (31), can also be easily checked.

Example 4 (Robust Quadratic Finite-time Stabilization) Let us study the robustness of the control system designed in Example 3 with the disturbance function

$$
\tilde{\omega}=\left(\begin{array}{c}
0 \\
\omega_{0} \\
0
\end{array}\right) .
$$

where the function $\omega_{0}$ is assumed to be bounded as follows

$$
\left|\omega_{0}\right| \leq \delta\|\xi\|_{d}, \quad \xi=\left(x_{1}, x_{2}, u\right)^{\top} .
$$

Taking into account $\|\xi\|_{\boldsymbol{d}}=\|z\|$ and $\boldsymbol{d}(s)=\operatorname{diag}\left\{e^{3 s}, e^{2 s}, e^{s}\right\}$ we derive

$$
\begin{gathered}
\sqrt{\tilde{\omega}^{\top} \boldsymbol{d}^{\top}(-\ln \|z\|) P \boldsymbol{d}(-\ln \|z\|) \tilde{\omega}}=\left|\omega_{0}\right| \sqrt{e_{2}^{\top} \boldsymbol{d}^{\top}(-\ln \|z\|) P \boldsymbol{d}(-\ln \|z\|) e_{2}}= \\
\frac{\left|\omega_{0}\right|}{\|z\|^{2}} \sqrt{e_{2}^{\top} P e_{2}} \leq \frac{\delta}{\|z\|} \sqrt{e_{2}^{\top} P e_{2}}
\end{gathered}
$$

Since $\operatorname{deg}_{\mathbb{F}_{d}}\left(f_{0}\right)=-1$ (see, Example 3) then the condition (32) becomes

$$
\frac{\delta}{\|z\|} \sqrt{e_{2}^{\top} P e_{2}} \leq-\frac{1}{\|z\|} \frac{z^{\top}}{\|z\|} \operatorname{Pf}\left(\frac{z}{\|z\|}\right) .
$$

and the sufficient condition of the robust finite-time stability is

$$
\delta \leq-\frac{\sup _{\|x\|=1} x^{\top} P f(x)}{\sqrt{e_{2}^{\top} P e_{2}}}, \quad e_{2}=(0,1,0)^{\top} \in \mathbb{R}^{3} .
$$

provided that $z^{\top} \operatorname{Pf}(z)<0$. 
Remark 4 The conditions (30) - (32) can be utilized for the robust global asymptotic stability analysis of an arbitrary system

$$
\dot{\sigma}(t)=f(\sigma(t))+\tilde{w}(t, \sigma(t)),
$$

where $f: \mathbb{R}^{n} \rightarrow \mathbb{R}$ is a d-homogeneous vector field and $\tilde{w}: \mathbb{R} \times \mathbb{R}^{n} \rightarrow \mathbb{R}^{n}$ is a disturbance. The well-known feature of the sliding mode systems is possible rejection of some bounded exogenous disturbances. The corresponding condition can be derived from (32) restricted as

$\sqrt{\tilde{\omega}^{\top} \boldsymbol{d}^{\top}(-\ln \|z\|) \operatorname{Pd}(-\ln \|z\|) \tilde{\omega}} \leq \delta\|z\|_{\boldsymbol{d}}^{\operatorname{deg}_{\mathbb{F}_{d}}(f)} \quad$ where $\quad \delta=-\sup _{\|z\|=1} z^{\top} \operatorname{Pf}(z)$.

The latter inequality holds, for example, if $G_{d}=\operatorname{diag}\{n, n-1, \ldots, 1\}, \operatorname{deg}_{\mathbb{F}_{d}}(f)=$ -1 and $\tilde{\omega}=\left(0,0, \ldots, 0, \tilde{\omega}_{n}\right)^{\top} \in \mathbb{R}^{n}$ with $\left|\tilde{\omega}_{n}\right| \leq \frac{\delta}{\sqrt{e_{n}^{\top} P e_{n}}}$.

\section{Discussions and Conclusions}

In the paper it is proved that any asymptotically stable generalized homogeneous system is equivalent (homeomorphic on $\mathbb{R}^{n}$ and diffeomorphic on $\mathbb{R}^{n} \backslash\{\mathbf{0}\}$ ) to a quadratically stable one. The necessary and sufficient condition of quadraticlike stability (stabilizability) of homogeneous (control) system is derived. This condition may simplify, in some particular cases (see, Section 4.1 and Examples 3,4 ) the design of finite-time stabilizing feedback laws (in particular, high order sliding mode control) and formulate an algorithm for control parameters tuning in terms of LMIs. Finally, robust stability/stabilizability conditions are derived as consequences of the obtained results. According to presented examples the obtained stability/stabilizability conditions can be useful for sliding mode control design in the case of negative homogeneity degree. In general case, an appropriate computational procedure is needed to be developed for quadratic-like Lyapunov function design (as well as for the canonical homogeneous norm calculation/estimation and control application) based on (16) (resp. (6) and (25)). This issue is considered as an open important problem for future research.

\section{References}

[1] D. J. Gross, "The role of symmetry in fundamental physics," Proceeding of the National Academy of Sciences of The United States of America, vol. 93, no. 25, pp. $14256-14259,1996$.

[2] V. Zubov, "On systems of ordinary differential equations with generalized homogenous right-hand sides," Izvestia vuzov. Mathematica., vol. 1, pp. 80-88, 1958 (in Russian). 
[3] H. Hermes, "Nilpotent approximations of control systems and distributions," SIAM Journal of Control and Optimization, vol. 24, no. 4, p. 731, 1986.

[4] M. Kawski, "Geometric homogeneity and stabilization," in Proc. IFAC Nonlinear Control Symposium, A. Krener and D. Mayne, Eds., Lake Tahoe, CA, 1995, pp. 164-169.

[5] L. Rosier, "Etude de quelques problemes de stabilization," PhD Thesis, Ecole Normale Superieure de Cachan (France), 1993.

[6] R. Sepulchre and D. Aeyels, "Homogeneous Lyapunov Functions and Necessary Conditions for Stabilization," Mathematics of Control, Signals and Systems, vol. 9, pp. 34-58, 1996.

[7] V. I. Zubov, Methods of A.M. Lyapunov and Their Applications. Noordhoff, Leiden, 1964.

[8] W. Hahn, Stability of Motion. New York: Springer-Verlag Berlin Heidelberg, 1967.

[9] A. Andreini, A. Bacciotti, and G. Stefani, "Global stabilizability of homogenenous vector fields of odd degree," Systems 83 Control Letters, vol. 10, pp. 251-256, 1988.

[10] J.-M. Coron and L. Praly, "Adding an integrator for the stabilization problem," Systems \& Control Letters, vol. 17, no. 2, pp. 89-104, 1991.

[11] Y. Orlov, "Finite time stability and robust control synthesis of uncertain switched systems," SIAM Journal of Control and Optimization, vol. 43, no. 4, pp. 1253-1271, 2005.

[12] A. Levant, "Homogeneity approach to high-order sliding mode design," Automatica, vol. 41, no. 5, pp. 823-830, 2005.

[13] L. Rosier, "Homogeneous Lyapunov function for homogeneous continuous vector field," Systems 83 Control Letters, vol. 19, pp. 467-473, 1992.

[14] W. Perruquetti, T. Floquet, and E. Moulay, "Finite-time observers: application to secure communication," IEEE Transactions on Automatic Control, vol. 53, no. 1, pp. 356-360, 2008.

[15] V. Andrieu, L. Praly, and A. Astolfi, "Homogeneous Approximation, Recursive Observer Design, and Output Feedback," SIAM Journal of Control and Optimization, vol. 47, no. 4, pp. 1814-1850, 2008.

[16] D. Efimov and W. Perruquetti, "Oscillations conditions in homogenous systems," in Proc. IFAC NOLCOS Symp., 2010, pp. 1379-1384. 
[17] A. Polyakov, D. Efimov, and W. Perruquetti, "Robust stabilization of mimo systems in finite/fixed time," International Journal of Robust and Nonlinear Control, vol. 26, no. 1, pp. 69-90, 2016.

[18] K. Zimenko, D. Efimov, A. Polyakov, and W. Perruquetti, "A note on delay robustness for homogeneous systems with negative degree," Automatica, vol. 79, no. 5, pp. 178-184, 2017.

[19] J.-B. Pomet and C. Samson, "Time-varying exponential stabilization of nonholonomic systems in power form," Inria, Tech. Rep., 1993.

[20] A. Polyakov, D. Efimov, E. Fridman, and W. Perruquetti, "On homogeneous distributed parameters equations," IEEE Transactions on Automatic Control, vol. 61, no. 11, pp. 3657-3662, 2016.

[21] V. V. Khomenuk, "On systems of ordinary differential equations with generalized homogenous right-hand sides," Izvestia vuzov. Mathematica., vol. 3(22), pp. 157-164, 1961 (in Russian).

[22] I. Karafyllis and Z.-P. Jiang, Stability and Stabilization of Nonlinear Systems. Springer, 2011.

[23] A. Poznyak, A. Polyakov, and V. Azhmyakov, Attractive Ellipsoids in Robust Control. Birkhauser, 2014.

[24] H. Hermes, "Homogeneous feedback controls for homogeneous systems," Systems $\&$ Control Letters, vol. 24, pp. 7-11, 1995.

[25] L. Praly, "Generalized weighted homogeneity and state dependent time scale for linear controllable systems," in Conference on Decision and Control, 1997, pp. 4342-4347.

[26] R. Sepulchre and D. Aeyels, "Stabilizability does not imply homogeneous stabilizability for controllable homogeneous systems," SIAM Journal of Control and Optimization, vol. 34, no. 5, pp. 1798-1813, 1996.

[27] L. Grune, "Homogeneous state feedback stabilization of homogeneous systems," SIAM Journal of Control and Optimization, vol. 38, no. 4, pp. 1288$1308,2000$.

[28] N. Nakamura, H. Nakamura, and Y. Yamashita, "Homogeneous stabilization for input-affine homogeneous systems," in Conference on Decision and Control, 2007, pp. 80-85.

[29] S. P. Bhat and D. S. Bernstein, "Geometric homogeneity with applications to finite-time stability," Mathematics of Control, Signals and Systems, vol. 17, pp. 101-127, 2005.

[30] E. Moulay and W. Perruquetti, "Finite-time stability of differential inclusions," IMA Journal Mathematical Control and Information Sciences, vol. 22(4), pp. 465-475, 2005. 
[31] V. I. Utkin, Sliding Modes in Control Optimization. Berlin: SpringerVerlag, 1992.

[32] S. Boyd, E. Ghaoui, E. Feron, and V. Balakrishnan, Linear Matrix Inequalities in System and Control Theory. Philadelphia: SIAM, 1994.

[33] Y. Nesterov and A. Nemirovskii, Interior Point Polynomial Algorithms in Convex Programming. SIAM (Studies in Appliad Mathematics), 1993.

[34] A. Polyakov, J.-M. Coron, and L. Rosier, "On finite-time stabilization of evolution equations: A homogeneous approach," in Conference on Decision and Control, 2016, pp. 3143-3148.

[35] A. Polyakov, "Quadratic-like stabilizability of homogeneous systems," in Conference on Decision and Control, (https://hal.inria.fr/hal-01525252), 2017.

[36] A. Levant, "Sliding order and sliding accuracy in sliding mode control," International Journal of Control, vol. 58, no. 6, pp. 1247-1263, 1993.

[37] A. Pazy, Semigroups of Linear Operators and Applications to Partial Differential Equations. Springer, 1983.

[38] A. Poznyak, Advanced Mathematical Tools for Automatic Control Engineers. Volume 1: Deterministic Technique. Elsevier, 2008.

[39] R. Courant and F. John, Introduction to calculus and analysis (Vol. II/1). New York: Springer, 2000.

[40] A. Bacciotti and L. Rosier, Lyapunov Functions and Stability in Control Theory. Springer, 2001.

[41] A. Polyakov, D. Efimov, and W. Perruquetti, "Finite-time and fixed-time stabilization: Implicit lyapunov function approach," Automatica, vol. 51, no. 1, pp. 332-340, 2015.

[42] A. Levant, "Quasi-continuous high-order sliding-mode controllers," IEEE Transactions on Automatic Control, vol. 50, no. 11, pp. 1812-1816, 2005.

[43] K. Zimenko, A. Polyakov, and D. Efimov, "Stabilization of chain of integrators with arbitrary in finite-time," in IEEE Conference on Decision and Control, 2015.

[44] E. Sontag, "A 'universal' construction of artstein's theorem on nonlinear stabilization," Systems \& Control Letters, vol. 13, pp. 117-123, 1989. 